\title{
Non-smooth atomic decomposition of variable 2-microlocal Besov-type and Triebel-Lizorkin-type spaces
}

\author{
Helena F. Gonçalves ${ }^{1}$ [D
}

Received: 16 November 2020 / Accepted: 8 April 2021 / Published online: 20 May 2021

(c) The Author(s) 2021

\begin{abstract}
In this paper we provide non-smooth atomic decompositions of 2-microlocal Besovtype and Triebel-Lizorkin-type spaces with variable exponents $B_{p(\cdot), q(\cdot)}^{\boldsymbol{w}, \phi}\left(\mathbb{R}^{n}\right)$ and $F_{p(\cdot), q(\cdot)}^{w, \phi}\left(\mathbb{R}^{n}\right)$. Of big importance in general, and an essential tool here, are the characterizations of the spaces via maximal functions and local means, that we also present. These spaces were recently introduced by $\mathrm{Wu}$ et al. and cover not only variable 2-microlocal Besov and Triebel-Lizorkin spaces $B_{p(\cdot), q(\cdot)}^{\boldsymbol{w}}\left(\mathbb{R}^{n}\right)$ and $F_{p(\cdot), q(\cdot)}^{\boldsymbol{w}}\left(\mathbb{R}^{n}\right)$, but also the more classical smoothness Morrey spaces $B_{p, q}^{s, \tau}\left(\mathbb{R}^{n}\right)$ and $F_{p, q}^{s, \tau}\left(\mathbb{R}^{n}\right)$. Afterwards, we state a pointwise multipliers assertion for this scale.
\end{abstract}

Keywords Besov-type spaces · Triebel-Lizorkin-type spaces · 2-microlocal spaces · Variable exponents · Atoms · Local means $\cdot$ Peetre maximal operator · Pointwise multipliers

Mathematics Subject Classification 42B25 · 42B35 · 46E35

\section{Introduction}

The introduction of function spaces with variable integrability, also known as variable exponent function spaces $L_{p(\cdot)}\left(\mathbb{R}^{n}\right)$, goes back to Orlicz [27] in 1931. However, only several decades later they were substantially studied, in the papers [21] of Kováčik and Rákosník, as well as [9] of Edmunds and Rákosník and [5] of Diening. The spaces $L_{p(\cdot)}\left(\mathbb{R}^{n}\right)$ have several applications, such as in fluid dynamics, image processing, PDEs and variational calculus. For an overview we refer to [7].

Communicated by Dachun Yang.

Helena F. Gonçalves

helena.goncalves@uni-jena.de

1 Institute of Mathematics, Friedrich-Schiller-University Jena, 07737 Jena, Germany 
The merger of the concepts of variable integrability and variable smoothness was done by Diening, Hästö and Roudenko in [6], where the authors defined Triebel-Lizorkin spaces with variable exponents $F_{p(\cdot), q(\cdot)}^{s(\cdot)}\left(\mathbb{R}^{n}\right)$. The interplay between the three parameters $s, q$ and $p$ can, easily and interestingly, be verified on the trace theorem on $\mathbb{R}^{n-1}$ proved by these authors [6, Theorem 3.13]. This interaction is also clear on the Sobolev embedding results obtained for these spaces by Vybíral [36].

For the Besov spaces it is not so easy to have also the parameter $q$ as a variable one. Almeida and Hästö introduced in [3] Besov spaces $B_{p(\cdot), q(\cdot)}^{s(\cdot)}\left(\mathbb{R}^{n}\right)$ with all three indices variable, using for that a different modular which already uses the variable structure on $q(\cdot)$. They proved the Sobolev and other usual embeddings in this scale.

A more general approach to spaces of variable smoothness are the so-called 2-microlocal function spaces, where the smoothness gets measured by a weight sequence $\boldsymbol{w}=\left(w_{j}\right)_{j \in \mathbb{N}_{0}}$. Besov spaces with such weight sequences appeared first in the works of Peetre [28] and Bony [4]. The variable 2-microlocal Besov and Triebel-Lizorkin spaces $B_{p(\cdot), q}^{w}\left(\mathbb{R}^{n}\right)$ and $F_{p(\cdot), q(\cdot)}^{w}\left(\mathbb{R}^{n}\right)$ were introduced by Kempka [16, 17]. Since then, several authors have devoted some attention to these spaces, expanding the knowledge about their properties. We mention $[1,2,10,11,13,14,18,19$, 23].

Function spaces with variable exponents represent a kind of approach that generalizes classical function spaces. However, there are different approaches, which also lead us to generalize Besov and Triebel-Lizorkin spaces. The Besov-type spaces $B_{p, q}^{s, \tau}\left(\mathbb{R}^{n}\right)$ and the Triebel-Lizorkin-type spaces $F_{p, q}^{s, \tau}\left(\mathbb{R}^{n}\right)$ are an example of that. They were introduced in [40] and, besides the classical Besov and Triebel-Lizorkin spaces, they also cover Triebel-Lizorkin-Morrey spaces introduced by Tang and Xu [31] and the hybrid functions spaces introduced and studied by Triebel in [33, 34], together with their use in heat and Navier-Stokes equations.

Recently also these scales got new variable versions. In [38, 39], the authors introduced Besov-type and Triebel-Lizorkin-type spaces with variable exponents $B_{p(\cdot), q(\cdot)}^{s(\cdot),}\left(\mathbb{R}^{n}\right)$ and $F_{p(\cdot), q(\cdot)}^{s(\cdot),}\left(\mathbb{R}^{n}\right)$, with $\phi$ being a measurable function on $\mathbb{R}_{+}^{n+1}$ which replaces the parameter $\tau$. Moreover, also the 2-microlocal versions $B_{p(\cdot), q(\cdot)}^{\boldsymbol{w}, \phi}\left(\mathbb{R}^{n}\right)$ and $F_{p(\cdot), q(\cdot)}^{w, \phi}\left(\mathbb{R}^{n}\right)$ were already introduced in [37]. Among other aspects, the authors characterized the spaces by means of atomic decompositions and obtained a trace result on hyperplanes. These spaces provide an unified approach that covers variable 2-microlocal Besov and Triebel-Lizorkin spaces $B_{p(\cdot), q(\cdot)}^{\boldsymbol{w}}\left(\mathbb{R}^{n}\right)$ and $F_{p(\cdot), q(\cdot)}^{\boldsymbol{w}}\left(\mathbb{R}^{n}\right)$, variable Besov-type and Triebel-Lizorkin-type spaces $B_{p(\cdot), q(\cdot)}^{s(\cdot), \phi}\left(\mathbb{R}^{n}\right)$ and $F_{p(\cdot), q(\cdot)}^{s(\cdot), \phi}\left(\mathbb{R}^{n}\right)$, and hence all the spaces that are already covered by these.

In this paper we aim to derive a non-smooth atomic characterization for the spaces $B_{p(\cdot), q(\cdot)}^{\boldsymbol{w}, \phi}\left(\mathbb{R}^{n}\right)$ and $F_{p(\cdot), q(\cdot)}^{\boldsymbol{w}, \phi}\left(\mathbb{R}^{n}\right)$. An essential tool here is their characterization via local means, which follows immediately from the characterization by maximal functions. Although this characterization was already considered in [37], now we prove a more general version, which is more in line with the results of this type present in the literature.

As for the characterization via non-smooth atoms, recently in [12] the authors proved a result for the spaces $F_{p(\cdot), q(\cdot)}^{s(\cdot),}\left(\mathbb{R}^{n}\right)$, which was the first result on this subject 
for this type of function spaces, even for the case of constant exponents. Now we extend it to the 2-microlocal spaces $F_{p(\cdot), q(\cdot)}^{w, \phi}\left(\mathbb{R}^{n}\right)$ and also complete this study by obtaining the counterpart for the Besov scale $B_{p(\cdot), q(\cdot)}^{w, \phi}\left(\mathbb{R}^{n}\right)$. Covered by these results will be also the results obtained in [10] for 2-microlocal variable Besov and Triebel-Lizorkin spaces.

Implicit in the name of this characterization-non-smooth - is the fact that we replace the usual (smooth) atoms by more general ones, in the sense that they have weaker assumptions on the smoothness. We then show that, also in this case, all the crucial information compared with smooth atomic decompositions is kept. This modification appeared first in [35], where Triebel and Winkelvoß suggested the use of these more relaxed conditions to define classical Besov and Triebel-Lizorkin spaces intrinsically on domains. More recent is the work [30] of Scharf, where a non-smooth atomic characterization for $B_{p, q}^{s}\left(\mathbb{R}^{n}\right)$ and $F_{p, q}^{s}\left(\mathbb{R}^{n}\right)$ was derived, using even more general atoms. Here we follow this approach to prove our main result. Moreover, as an application, we provide an assertion on pointwise multipliers for the spaces $B_{p(\cdot), q(\cdot)}^{\boldsymbol{w}, \phi}\left(\mathbb{R}^{n}\right)$ and $F_{p(\cdot), q(\cdot)}^{\boldsymbol{w}, \phi}\left(\mathbb{R}^{n}\right)$.

\section{Notation and definitions}

We start by collecting some general notation used throughout the paper.

As usual, we denote by $\mathbb{N}$ the set of all natural numbers, $\mathbb{N}_{0}=\mathbb{N} \cup\{0\}$, and $\mathbb{R}^{n}$, $n \in \mathbb{N}$, the $n$-dimensional real Euclidean space with $|x|$, for $x \in \mathbb{R}^{n}$, denoting the Euclidean norm of $x$. By $\mathbb{Z}^{n}$ we denote the lattice of all points in $\mathbb{R}^{n}$ with integer components. For $\beta:=\left(\beta_{1}, \cdots, \beta_{n}\right) \in \mathbb{Z}^{n}$, let $|\beta|:=\left|\beta_{1}\right|+\cdots+\left|\beta_{n}\right|$. If $a, b \in \mathbb{R}$, then $(a \vee b):=\max \{a, b\}$.

We denote by $c$ a generic positive constant which is independent of the main parameters, but its value may change from line to line. The expression $A \lesssim B$ means that $A \leq c B$. If $A \lesssim B$ and $B \lesssim A$, then we write $A \sim B$.

Given two quasi-Banach spaces $X$ and $Y$, we write $X \hookrightarrow Y$ if $X \subset Y$ and the natural embedding is bounded.

If $E$ is a measurable subset of $\mathbb{R}^{n}$, we denote by $\chi_{E}$ its characteristic function and by $|E|$ its Lebesgue measure. By supp $f$ we denote the support of the function $f$.

For each cube $Q \subset \mathbb{R}^{n}$ we denote its center by $c_{Q}$ and its side length by $\ell(Q)$. Moreover, for $a \in(0, \infty)$ we denote by $a Q$ the cube concentric with $Q$ having the side length $a \ell(Q)$. For $x \in \mathbb{R}^{n}$ and $r \in(0, \infty), Q(x, r)$ stands for the cube centered at $x$ with side length $r$, whose sides are parallel to the axes of coordinates.

Given $k \in \mathbb{N}_{0}, C^{k}\left(\mathbb{R}^{n}\right)$ is the space of all functions $f: \mathbb{R}^{n} \rightarrow \mathbb{C}$ which are $k$-times continuously differentiable (continuous in $k=0$ ) such that

$$
\left\|f\left|C^{k}\left(\mathbb{R}^{n}\right) \|:=\sum_{|\alpha| \leq k} \sup _{x \in \mathbb{R}^{n}}\right| D^{\alpha} f(x) \mid<\infty .\right.
$$

The Hölder space $\mathscr{C}\left(\mathbb{R}^{n}\right)$ with index $s>0$ is defined as the set of all functions $f \in C^{\lfloor s\rfloor^{-}}\left(\mathbb{R}^{n}\right)$ with 


$$
\begin{aligned}
\left\|f \mid \mathscr{C}^{S}\left(\mathbb{R}^{n}\right)\right\|:= & \left\|f \mid C^{\lfloor s\rfloor^{-}}\left(\mathbb{R}^{n}\right)\right\| \\
& +\sum_{|\alpha|=\{s\}^{+}} \sup _{y \in \mathbb{R}^{n}, x \neq y} \frac{\left|D^{\alpha} f(x)-D^{\alpha} f(y)\right|}{|x-y|^{\{s\}^{+}}}<\infty,
\end{aligned}
$$

where $\lfloor s\rfloor^{-} \in \mathbb{N}_{0}$ and $\{s\}^{+} \in(0,1]$ are uniquely determined numbers so that $s=\lfloor s\rfloor^{-}+\{s\}^{+}$. If $s=0$ we set $\mathscr{C}\left(\mathbb{R}^{n}\right):=L_{\infty}\left(\mathbb{R}^{n}\right)$.

By $\mathscr{S}\left(\mathbb{R}^{n}\right)$ we denote the usual Schwartz class of all infinitely differentiable rapidly decreasing complex-valued functions on $\mathbb{R}^{n}$ and $\mathscr{S}\left(\mathbb{R}^{n}\right)$ stands for the dual space of tempered distributions. The Fourier transform of $f \in \mathscr{S}\left(\mathbb{R}^{n}\right)$ or $f \in \mathscr{S}\left(\mathbb{R}^{n}\right)$ is denoted by $\widehat{f}$, while its inverse transform is denoted by $f^{\vee}$.

Now we give a short survey on variable exponents. By $\mathcal{P}\left(\mathbb{R}^{n}\right)$ we denote the set of all measurable functions $p: \mathbb{R}^{n} \rightarrow(0, \infty]$ (called variable exponents) which are essentially bounded away from zero. For $p \in \mathcal{P}\left(\mathbb{R}^{n}\right)$, we denote

$$
p^{-}:=\underset{x \in \mathbb{R}^{n}}{\operatorname{essinf}} p(x) \quad \text { and } \quad p^{+}:=\underset{x \in \mathbb{R}^{n}}{\operatorname{ess} \sup } p(x) .
$$

The variable exponent Lebesgue space $L_{p(\cdot)}(E)$ is the set of all (complex or realvalued) measurable functions $f$ such that

$$
\left\|f \mid L_{p(\cdot)}(E)\right\|:=\inf \left\{\lambda \in(0, \infty): \varrho_{p(\cdot)}\left(\frac{f \chi_{E}}{\lambda}\right) \leq 1\right\}<\infty .
$$

Here, $\varrho_{p(\cdot)}$ denotes the semimodular defined by

$$
\varrho_{p(\cdot)}(f):=\int_{\mathbb{R}^{n}} \phi_{p(x)}(|f(x)|) d x,
$$

where

$$
\phi_{p(x)}(t):= \begin{cases}t^{p(x)} & \text { if } p(x) \in(0, \infty), \\ 0 & \text { if } p(x)=\infty \text { and } t \in[0,1], \\ \infty & \text { if } p(x)=\infty \text { and } t \in(1, \infty] .\end{cases}
$$

It is known that $L_{p(\cdot)}(E)$ is a quasi-Banach space and becomes a Banach space when $p^{-} \geq 1$. As we will need it later, let us recall that $L_{p(\cdot)}(E)$ has the lattice property. Moreover, it is easy to verify that

$$
\left\|\left.f\left|L_{p(\cdot)}(E)\|=\|\right| f\right|^{r} \mid L_{\frac{p(\cdot)}{r}}(E)\right\|^{\frac{1}{r}}, \quad r \in(0, \infty) .
$$

If $p(\cdot) \equiv p$ is constant, then $L_{p(\cdot)}(E)=L_{p}(E)$ is the classical Lebesgue space.

\subsection{Mixed sequence-Lebesgue spaces}

We recall now mixed sequence-Lebesgue spaces and, in the next section, we present some properties about them, which will be very useful throughout this work. 
Definition 2.1 Let $p, q \in \mathcal{P}\left(\mathbb{R}^{n}\right)$ and $E$ be a measurable subset of $\mathbb{R}^{n}$.

(i) Let $p^{+}, q^{+}<\infty$. The space $L_{p(\cdot)}\left(\ell_{q(\cdot)}(E)\right)$ is defined to be the set of all sequences of measurable functions $\left(f_{j}\right)_{j \in \mathbb{N}_{0}}$ on $E$ such that

$$
\left\|\left(f_{j}\right)_{j \in \mathbb{N}_{0}}\left|L_{p(\cdot)}\left(\ell_{q(\cdot)}(E)\right)\|:=\|\left(\sum_{j=0}^{\infty}\left|f_{j}(x)\right|^{q(x)}\right)^{1 / q(x)}\right| L_{p(\cdot)}(E)\right\|<\infty .
$$

(ii) The space $\ell_{q(\cdot)}\left(L_{p(\cdot)}(E)\right)$ is defined to be the set of all sequences of measurable functions $\left(f_{j}\right)_{j \in \mathbb{N}_{0}}$ on $E$ such that

$$
\begin{aligned}
& \left\|\left(f_{j}\right)_{j \in \mathbb{N}_{0}} \mid \ell_{q(\cdot)}\left(L_{p(\cdot)}(E)\right)\right\| \\
& \quad:=\inf \left\{\mu>0: \varrho_{\ell_{q(\cdot)}\left(L_{p(\cdot)}\right)}\left(\left(\frac{f_{j} \chi_{E}}{\mu}\right)_{j \in \mathbb{N}_{0}}\right) \leq 1\right\}<\infty,
\end{aligned}
$$

where, for all sequences $\left(g_{j}\right)_{j \in \mathbb{N}_{0}}$ of measurable functions,

$\varrho_{\ell_{q(\cdot)}\left(L_{p(\cdot)}\right)}\left(\left(g_{j}\right)_{j \in \mathbb{N}_{0}}\right):=\sum_{j=0}^{\infty} \inf \left\{\lambda_{j}>0: \varrho_{p(\cdot)}\left(\frac{g_{j}}{\lambda_{j}^{1 / q(\cdot)}}\right) \leq 1\right\}$,

with the convention $\lambda^{1 / \infty}=1$ for all $\lambda \in(0, \infty)$.

\section{Remark 2.2}

(i) If $E=\mathbb{R}^{n}$, we simply write $L_{p(\cdot)}\left(\ell_{q(\cdot)}\right)$ or $\ell_{q(\cdot)}\left(L_{p(\cdot)}\right)$.

(ii) If $p, q \in \mathcal{P}\left(\mathbb{R}^{n}\right)$, then $\left\|\cdot \mid L_{p(\cdot)}\left(\ell_{q(\cdot)}\right)\right\|$ and $\left\|\cdot \mid \ell_{q(\cdot)}\left(L_{p(\cdot)}\right)\right\|$ are quasi-norms in $L_{p(\cdot)}\left(\ell_{q(\cdot)}\right)$ and $\ell_{q(\cdot)}\left(L_{p(\cdot)}\right)$, respectively. Moreover, $\left\|\cdot \mid L_{p(\cdot)}\left(\ell_{q(\cdot)}\right)\right\|$ is a norm if $\min \left\{p^{-}, q^{-}\right\} \geq 1$. The same does not hold when it comes to the norm in (2.2). It was shown in [3] that $\left\|\cdot \mid \ell_{q(\cdot)}\left(L_{p(\cdot)}\right)\right\|$ is a norm either when $q \geq 1$ is constant and $p^{-} \geq 1$, or when $\frac{1}{p(x)}+\frac{1}{q(x)} \leq 1$ almost everywhere. More recently, it was proved in [20] that it also becomes a norm if $1 \leq q(x) \leq p(x) \leq \infty$.

(iii) It was proved in [3, Theorem 3.5] that $\varrho_{\ell_{q(\cdot)}\left(L_{p(\cdot)}\right)}$ is a semi-modular. The leftcontinuity property ensures that the unit ball property holds, i.e.,

$\left\|\left(f_{j}\right)_{j \in \mathbb{N}_{0}} \mid \ell_{q(\cdot)}\left(L_{p(\cdot)}\right)\right\| \leq 1 \quad$ if, and only if, $\quad \varrho_{\ell_{q(\cdot)}\left(L_{p(\cdot)}\right)}\left(\left(f_{j}\right)_{j \in \mathbb{N}_{0}}\right) \leq 1$.

Moreover, for $r \in(0, \infty)$,

$$
\left\|( f _ { j } ) _ { j \in \mathbb { N } _ { 0 } } | \ell _ { q ( \cdot ) } ( L _ { p ( \cdot ) } ) \| = \| ( | f _ { j } | ^ { r } ) _ { j \in \mathbb { N } _ { 0 } } | \ell _ { \frac { q ( \cdot ) } { r } } \left(\frac{\left.L_{\frac{p(\cdot)}{r}}\right)}{1 / r} \|^{1 / r} .\right.\right.
$$

(iv) If $q^{+}<\infty$, then we can replace (2.3) by the simpler expression 


$$
\varrho_{\ell_{q(\cdot)}\left(L_{p(\cdot)}\right)}\left(\left(g_{j}\right)_{j \in \mathbb{N}_{0}}\right)=\sum_{j=0}^{\infty}\left\|\left|g_{j}\right|^{q(\cdot)} \mid L_{\frac{p(\cdot)}{q(\cdot)}}\left(\mathbb{R}^{n}\right)\right\| .
$$

Let $\mathcal{G}\left(\mathbb{R}_{+}^{n+1}\right)$ be the set of all measurable functions $\phi: \mathbb{R}^{n} \times[0, \infty) \rightarrow(0, \infty)$ having the following properties: there exist positive constants $c_{1}(\phi)$ and $\tilde{c_{1}}(\phi)$ such that

$$
\frac{1}{\tilde{c_{1}}(\phi)} \leq \frac{\phi(x, r)}{\phi(x, 2 r)} \leq c_{1}(\phi) \quad \text { for all } x \in \mathbb{R}^{n} \text { and } r \in(0, \infty),
$$

and there exists a positive constant $c_{2}(\phi)$ such that, for all $x, y \in \mathbb{R}^{n}$ and $r \in(0, \infty)$ with $|x-y| \leq r$,

$$
\frac{1}{c_{2}(\phi)} \leq \frac{\phi(x, r)}{\phi(y, r)} \leq c_{2}(\phi) .
$$

The conditions (2.5) and (2.6) are called doubling condition and compatibility condition, respectively, and have been used by Nakai [24, 25] and Nakai and Sawano [26]. Examples of functions in $\mathcal{G}\left(\mathbb{R}_{+}^{n+1}\right)$ are provided in [38, Remark 1.3].

In what follows, for $\phi \in \mathcal{G}\left(\mathbb{R}_{+}^{n+1}\right)$ and a cube $Q:=Q(x, r)$ with center $x \in \mathbb{R}^{n}$ and side length $r \in(0, \infty)$, we define $\phi(Q):=\phi(Q(x, r)):=\phi(x, r)$. The following is our convention for dyadic cubes: For $j \in \mathbb{Z}$ and $k \in \mathbb{Z}^{n}$, denote by $Q_{j k}$ the dyadic cube $2^{-j}\left([0,1)^{n}+k\right)$ and $x_{Q_{j k}}$ its lower left corner. Let $\mathcal{Q}:=\left\{Q_{j k}: j \in \mathbb{Z}, k \in \mathbb{Z}^{n}\right\}$, $\mathcal{Q}^{*}:=\{Q \in \mathcal{Q}: \ell(Q) \leq 1\}$ and $j_{Q}:=-\log _{2} \ell(Q)$ for all $Q \in \mathcal{Q}$. When the dyadic cube $Q$ appears as an index, such as $\sum_{Q \in \mathcal{Q}}$ and $(\cdots)_{Q \in \mathcal{Q}}$, it is understood that $Q$ runs over all dyadic cubes in $\mathbb{R}^{n}$.

For the function spaces under consideration in this paper, the following modified mixed-Lebesgue sequence spaces are of special importance.

Definition 2.3 Let $p, q \in \mathcal{P}\left(\mathbb{R}^{n}\right)$ and $\phi \in \mathcal{G}\left(\mathbb{R}_{+}^{n+1}\right)$.

(i) We denote by $\ell_{q(\cdot)}^{\phi}\left(L_{p(\cdot)}\right)$ the set of all sequences $\left(g_{j}\right)_{j \in \mathbb{N}_{0}}$ of measurable functions on $\mathbb{R}^{n}$ such that

$$
\left\|\left(g_{j}\right)_{j \in \mathbb{N}_{0}}\left|\ell_{q(\cdot)}^{\phi}\left(L_{p(\cdot)}\right)\left\|:=\sup _{P \in \mathcal{Q}} \frac{1}{\phi(P)}\right\|\left(g_{j}\right)_{j \geq\left(j_{p} \vee 0\right)}\right| \ell_{q(\cdot)}\left(L_{p(\cdot)}(P)\right)\right\|<\infty
$$

where the supremum is taken over all dyadic cubes $P$ in $\mathbb{R}^{n}$.

(ii) We denote by $L_{p(\cdot)}^{\phi}\left(\ell_{q(\cdot)}\right)$ the set of all sequences $\left(g_{j}\right)_{j \in \mathbb{N}_{0}}$ of measurable functions on $\mathbb{R}^{n}$ such that

$$
\left\|\left(g_{j}\right)_{j \in \mathbb{N}_{0}}\left|L_{p(\cdot)}^{\phi}\left(\ell_{q(\cdot)}\right)\left\|:=\sup _{P \in \mathcal{Q}} \frac{1}{\phi(P)}\right\|\left(g_{j}\right)_{j \geq\left(j_{p} \vee 0\right)}\right| L_{p(\cdot)}\left(\ell_{q(\cdot)}(P)\right)\right\|<\infty
$$

where the supremum is taken over all dyadic cubes $P$ in $\mathbb{R}^{n}$. 


\section{Remark 2.4}

(i) We remark that $\ell_{q(\cdot)}^{\phi}\left(L_{p(\cdot)}\right)$ and $L_{p(\cdot)}^{\phi}\left(\ell_{q(\cdot)}\right)$ are quasi-normed spaces that coincide with the mixed Lebesgue-sequence spaces $\ell_{q(\cdot)}\left(L_{p(\cdot)}\right)$ and $L_{p(\cdot)}\left(\ell_{q(\cdot)}\right)$ from Definition 2.1, respectively, when $\phi \equiv 1$. The case of $q(\cdot)=q$ constant and $\phi(Q):=|Q|^{\tau}$ for all cubes $Q$ and $\tau \in[0, \infty)$, has also been considered in [22].

(ii) If we replace the dyadic cubes in the Definition 2.3 by arbitrary cubes $P$, then we obtain equivalent quasi-norms.

\subsection{Auxiliary results}

Although the Hardy-Littlewood maximal operator $\mathcal{M}_{t}$ constitutes a great tool in the theory of classical function spaces and also in the scale of variable Lebesgue spaces, it is not, in general, a good instrument in the mixed spaces $L_{p(\cdot)}\left(\ell_{q(\cdot)}\right)$ and $\ell_{q(\cdot)}\left(L_{p(\cdot)}\right)$. It was actually proved in [3] and in [6] that this operator is not bounded in these spaces if one considers $q$ non-constant. However, this adversity can be overcome by the use of convolution inequalities involving radially decreasing kernels, namely the so-called $\eta$-functions, defined by

$$
\eta_{\nu, R}(x):=\frac{2^{n v}}{\left(1+2^{v}|x|\right)^{R}}, \quad x \in \mathbb{R}^{n},
$$

for $\nu \in \mathbb{N}_{0}$ and $R>0$.

Let us start by recalling important classes related to the regularity of the exponents.

Definition 2.5 Let $g \in C\left(\mathbb{R}^{n}\right)$. We say that $g$ is locally log-Hölder continuous, abbreviated $g \in C_{\operatorname{loc}}^{\log }\left(\mathbb{R}^{n}\right)$, if there exists $c_{\log }(g)>0$ such that

$$
|g(x)-g(y)| \leq \frac{c_{\log }(g)}{\log (e+1 /|x-y|)} \quad \text { for all } x, y \in \mathbb{R}^{n} .
$$

We say that $g$ is globally log-Hölder continuous, abbreviated $g \in C^{\log }\left(\mathbb{R}^{n}\right)$, if $g$ is locally log-Hölder continuous and there exists $g_{\infty} \in \mathbb{R}$ such that

$$
\left|g(x)-g_{\infty}\right| \leq \frac{c_{\log }}{\log (e+|x|)} \quad \text { for all } x \in \mathbb{R}^{n} .
$$

Note that all functions in $C_{\operatorname{loc}}^{\log }\left(\mathbb{R}^{n}\right)$ are bounded and if $g \in C^{\log }\left(\mathbb{R}^{n}\right)$ then $g_{\infty}=\lim _{|x| \rightarrow \infty} g(x)$. Moreover, for $g \in \mathcal{P}\left(\mathbb{R}^{n}\right)$ with $g_{+}<\infty$, we have that $g \in C^{\log }\left(\mathbb{R}^{n}\right)$ if, and only if, $1 / g \in C^{\log }\left(\mathbb{R}^{n}\right)$. The notation $\mathcal{P}^{\log }\left(\mathbb{R}^{n}\right)$ is used for those variable exponents $p \in \mathcal{P}\left(\mathbb{R}^{n}\right)$ with $p \in C^{\log }\left(\mathbb{R}^{n}\right)$.

The next result was proved in [7, Lemma 4.6.3] and shows that the convolution operator is well-behaved in $L_{p(\cdot)}\left(\mathbb{R}^{n}\right)$ for $p \in \mathcal{P}^{\log }\left(\mathbb{R}^{n}\right)$, when considering radially decreasing integrable functions. 
Lemma 2.6 Let $\quad p \in \mathcal{P}^{\log }\left(\mathbb{R}^{n}\right) \quad$ with $\quad p(x) \geq 1$. Let $\quad \psi \in L_{1}\left(\mathbb{R}^{n}\right) \quad$ and $\psi_{\varepsilon}(x):=\varepsilon^{-n} \psi(x / \varepsilon)$, for $\varepsilon>0$. Suppose that $\Psi(x):=\sup _{|y| \geq|x|}|\psi(y)|$ is integrable and $f \in L_{p(\cdot)}\left(\mathbb{R}^{n}\right)$. Then

$$
\left\|\psi_{\varepsilon} * f\left|L_{p(\cdot)}\left(\mathbb{R}^{n}\right)\|\lesssim\| \Psi\right| L_{1}\left(\mathbb{R}^{n}\right)\right\|\left\|f \mid L_{p(\cdot)}\left(\mathbb{R}^{n}\right)\right\|,
$$

where the implicit constant depends only on $n$ and $p$.

Remark 2.7 Note that we can use the previous lemma for the $\eta$-functions defined above. Namely, taking $\psi=\eta_{0, m}$ with $m>n$, then we have $\Psi=\eta_{0, m} \in L_{1}\left(\mathbb{R}^{n}\right)$. Thus, setting $\psi_{\varepsilon}=\eta_{v, m}$ with $\varepsilon=2^{-v}$, we get

$$
\left\|\eta_{v, m} * f\left|L_{p(\cdot)}\left(\mathbb{R}^{n}\right)\|\lesssim\| f\right| L_{p(\cdot)}\left(\mathbb{R}^{n}\right)\right\|
$$

if $m>n$, for $f \in L_{p(\cdot)}\left(\mathbb{R}^{n}\right)$ and $p \in \mathcal{P}^{\log }\left(\mathbb{R}^{n}\right)$ with $p(x) \geq 1$, for $x \in \mathbb{R}^{n}$.

The following two results show that the $\eta$-functions are well suited for the mixed Lebesgue-sequence spaces. The first one was proved in [6, Theorem 3.2] and the second goes back to [19, Lemma 10].

Lemma 2.8 Let $p, q \in \mathcal{P}^{\log }\left(\mathbb{R}^{n}\right)$ with $1<p^{-} \leq p^{+}<\infty$ and $1<q^{-} \leq q^{+}<\infty$. Then there exists a constant $c>0$ such that the inequality

$$
\left\|\left(\eta_{\nu, R} * f_{v}\right)_{v \in \mathbb{N}_{0}}\left|L_{p(\cdot)}\left(\ell_{q(\cdot)}\right)\|\leq c\|\left(f_{v}\right)_{v \in \mathbb{N}_{0}}\right| L_{p(\cdot)}\left(\ell_{q(\cdot)}\right)\right\|
$$

holds for every sequence $\left(f_{v}\right)_{v \in \mathbb{N}_{0}}$ of $L_{1}^{\mathrm{loc}}\left(\mathbb{R}^{n}\right)$ functions and constant $R>n$.

Lemma 2.9 Let $p, q \in \mathcal{P}^{\log }\left(\mathbb{R}^{n}\right)$ with $p^{-} \geq 1$. For all $R>n+c_{\log }(1 / q)$, there exists a constant $c>0$ such that for all sequences $\left(f_{v}\right)_{v \in \mathbb{N}_{0}} \in \ell_{q(\cdot)}\left(L_{p(\cdot)}\right)$ it holds

$$
\left\|\left(\eta_{v, R} * f_{v}\right)_{v \in \mathbb{N}_{0}}\left|\ell_{q(\cdot)}\left(L_{p(\cdot)}\right)\|\leq c\|\left(f_{v}\right)_{v \in \mathbb{N}_{0}}\right| \ell_{q(\cdot)}\left(L_{p(\cdot)}\right)\right\| .
$$

In the next result we state the corresponding counterparts for the modified mixed Lebesgue sequence spaces from Definition 2.3. We make use of the important fact that the supremums in this definition can equivalently be taken over all cubes in $\mathbb{R}^{n}$, as stated in Remark 2.4(ii).

Lemma 2.10 Let $p, q \in \mathcal{P}^{\log }\left(\mathbb{R}^{n}\right)$ and $\phi \in \mathcal{G}\left(\mathbb{R}_{+}^{n+1}\right)$.

(i) Let $p^{-} \geq 1$. If

$$
R>n+c_{\log }(1 / q)+\max \left\{0, \log _{2} \tilde{c}_{1}(\phi)\right\},
$$

then there exists $c>0$ such that for all sequences $\left(f_{v}\right)_{v \in \mathbb{N}_{0}} \in \ell_{q(\cdot)}^{\phi}\left(L_{p(\cdot)}\right)$ it holds 


$$
\left\|\left(\eta_{v, R} * f_{v}\right)_{v \in \mathbb{N}_{0}}\left|\ell_{q(\cdot)}^{\phi}\left(L_{p(\cdot)}\right)\|\leq c\|\left(f_{v}\right)_{v \in \mathbb{N}_{0}}\right| \ell_{q(\cdot)}^{\phi}\left(L_{p(\cdot)}\right)\right\|
$$

(ii) Let $1 \leq p^{-} \leq p^{+}<\infty$ and $1 \leq q^{-} \leq q^{+}<\infty$. If

$$
R>n+\max \left\{0, \log _{2} \tilde{c}_{1}(\phi)\right\}
$$

then there exists $c>0$ such that for all sequences $\left(f_{v}\right)_{v \in \mathbb{N}_{0}} \in L_{p(\cdot)}^{\phi}\left(\ell_{q(\cdot)}\right)$ it holds

$$
\left\|\left(\eta_{v, R} * f_{v}\right)_{v \in \mathbb{N}_{0}}\left|L_{p(\cdot)}^{\phi}\left(\ell_{q(\cdot)}\right)\|\leq c\|\left(f_{v}\right)_{v \in \mathbb{N}_{0}}\right| L_{p(\cdot)}^{\phi}\left(\ell_{q(\cdot)}\right)\right\| .
$$

Proof We will prove part (i), as the second follows similarly.

For any given dyadic cube $P \in \mathcal{Q}$ and any $v \in \mathbb{N}_{0}$, we decompose each $f_{v}$ into the sum

$$
f_{v}=f_{v}^{0}+\sum_{i=1}^{\infty} f_{v}^{i}
$$

where

$$
f_{v}^{0}:=f_{v} \chi_{Q\left(c_{P}, 2^{-j_{P}+1}\right)}
$$

and

$$
f_{v}^{i}:=f_{v} \chi_{R_{i}}, \quad \text { with } \quad R_{i}:=Q\left(c_{P}, 2^{-j_{P}+i+1}\right) \backslash Q\left(c_{P}, 2^{-j_{P}+i}\right),
$$

for $i \in \mathbb{N}$ and $c_{P}$ being the center of the cube $P$. Then we have

$$
\begin{aligned}
& \frac{1}{\phi(P)}\left\|\left(\eta_{v, R} * f_{v}\right)_{v \geq\left(j_{P} \vee 0\right)} \mid \ell_{q(\cdot)}\left(L_{p(\cdot)}(P)\right)\right\| \\
& \leq \frac{1}{\phi(P)}\left\|\left(\eta_{v, R} * f_{v}^{0}\right)_{v \geq\left(j_{P} \vee 0\right)} \mid \ell_{q(\cdot)}\left(L_{p(\cdot)}(P)\right)\right\| \\
& \quad+\frac{1}{\phi(P)}\left\|\left(\sum_{i=1}^{\infty} \eta_{v, R} * f_{v}^{i}\right)_{v \geq\left(j_{P} \vee 0\right)} \mid \ell_{q(\cdot)}\left(L_{p(\cdot)}(P)\right)\right\| \\
& =: I_{1}+I_{2} .
\end{aligned}
$$

We claim that, for $j=1,2$,

$$
I_{j} \lesssim\left\|\left(f_{v}\right)_{v \in \mathbb{N}_{0}} \mid \ell_{q(\cdot)}^{\phi}\left(L_{p(\cdot)}\right)\right\|,
$$

which, together with the arbitrariness of $P \in \mathcal{Q}$, allows us to conclude the proof.

Step 1. Let us prove (2.9) for $j=1$. Here we apply Lemma 2.9 with $R>n+c_{\log }(1 / q)$ and use (2.5) to obtain 


$$
\begin{aligned}
I_{1} & =\frac{1}{\phi(P)}\left\|\left(\eta_{v, R} * f_{v}^{0}\right)_{v \geq\left(j_{P} \vee 0\right)} \mid \ell_{q(\cdot)}\left(L_{p(\cdot)}(P)\right)\right\| \\
& \leq \frac{1}{\phi(P)}\left\|\left(f_{v}^{0}\right)_{v \geq\left(j_{P} \vee 0\right)} \mid \ell_{q(\cdot)}\left(L_{p(\cdot)}\left(\mathbb{R}^{n}\right)\right)\right\| \\
& \leq \tilde{c}_{1}(\phi) \frac{1}{\phi\left(Q\left(c_{P}, 2^{-j_{p}+1}\right)\right)}\left\|\left(f_{\nu} \chi_{Q\left(c_{P}, 2^{-j_{P}+1}\right)}\right)_{v \geq\left(j_{P} \vee 0\right)} \mid \ell_{q(\cdot)}\left(L_{p(\cdot)}\left(\mathbb{R}^{n}\right)\right)\right\| \\
& \lesssim\left\|\left(f_{v}\right)_{v \in \mathbb{N}_{0}} \mid \ell_{q(\cdot)}^{\phi}\left(L_{p(\cdot)}\right)\right\|,
\end{aligned}
$$

as we desired.

Step 2. To estimate $I_{2}$, we start by estimating the convolution appearing here. Note that, for $x \in P, y \in R_{i}, i \in \mathbb{N}$, and $v \geq j_{P}$, we have $|x-y| \geq 2^{i-1-j_{P}} \geq 2^{i-1-v}$ and hence

$$
\left(\eta_{\nu, R} * f_{v}^{i}\right)(x)=\int_{\mathbb{R}^{n}} \frac{2^{v n}}{\left(1+2^{v}|x-y|\right)^{R}} f_{v}^{i}(y) d y \lesssim 2^{-i \varepsilon}\left(\eta_{v, R-\varepsilon} * f_{v}^{i}\right)(x),
$$

for some constant $\varepsilon$ satisfying $\varepsilon>\max \left\{0, \log _{2} \tilde{c}_{1}(\phi)\right\}$ and $R-\varepsilon>n+c_{\log }(1 / q)$. Therefore, using this, Lemma 2.9 and (2.6), we get

$$
\begin{aligned}
I_{2} & \lesssim \frac{1}{\phi(P)} \sum_{i=1}^{\infty} 2^{-i \varepsilon}\left\|\left(\eta_{v, R-\varepsilon} * f_{v}^{i}\right)_{v \geq\left(j_{P} \vee 0\right)} \mid \ell_{q(\cdot)}\left(L_{p(\cdot)}(P)\right)\right\| \\
& \leq \frac{1}{\phi(P)} \sum_{i=1}^{\infty} 2^{-i \varepsilon}\left\|\left(f_{v}^{i}\right)_{v \geq\left(j_{P} \vee 0\right)} \mid \ell_{q(\cdot)}\left(L_{p(\cdot)}\left(\mathbb{R}^{n}\right)\right)\right\| \\
& \leq \sum_{i=1}^{\infty} 2^{-i \varepsilon} \frac{\phi\left(Q\left(c_{P}, 2^{-j_{p}+i+1}\right)\right)}{\phi(P)}\left\|\left(f_{v}\right)_{v \in \mathbb{N}_{0}} \mid \ell_{q(\cdot)}^{\phi}\left(L_{p(\cdot)}\right)\right\| \\
& \leq\left\|\left(f_{v}\right)_{v \in \mathbb{N}_{0}} \mid \ell_{q(\cdot)}^{\phi}\left(L_{p(\cdot)}\right)\right\| \sum_{i=1}^{\infty} 2^{-i\left(\varepsilon-\log _{2} \tilde{c}_{1}(\phi)\right)} \\
& \lesssim\left\|\left(f_{v}\right)_{v \in \mathbb{N}_{0}} \mid \ell_{q(\cdot)}^{\phi}\left(L_{p(\cdot)}\right)\right\|,
\end{aligned}
$$

which allows us to conclude the proof of (2.9). Consequently the proof of Lemma 2.10 is complete.

Remark 2.11 In [42, Lemma 2.2] and [37, Lemma 3.12] the authors stated a similar result, but with a stronger condition on the parameter $R$. Using a different decomposition of each function $f_{v}$, we obtained here an extended version of those results.

Lastly, we present a discrete convolution inequality, which extends [37, Lemma 5.6] slightly.

Lemma 2.12 Let $p, q \in \mathcal{P}\left(\mathbb{R}^{n}\right)$ and $\phi \in \mathcal{G}\left(\mathbb{R}_{+}^{n+1}\right)$. Let $D_{1}, D_{2} \in(0, \infty)$ with $D_{2}>\max \left\{0, \log _{2} \tilde{c}_{1}(\phi)\right\}$. For any sequence $\left(g_{v}\right)_{v \in \mathbb{N}_{0}}$ of measurable functions on $\mathbb{R}^{n}$, consider 


$$
G_{j}(x):=\sum_{\nu=0}^{j} 2^{-(j-v) D_{2}} g_{\nu}(x)+\sum_{\nu=j+1}^{\infty} 2^{-(\nu-j) D_{1}} g_{\nu}(x), \quad x \in \mathbb{R}^{n}, \quad j \in \mathbb{N}_{0} .
$$

Then there exist constants $C_{1}, C_{2}>0$, depending on $p(\cdot), q(\cdot)$ and $\phi$, such that

$$
\left\|\left(G_{j}\right)_{j \in \mathbb{N}_{0}}\left|\ell_{q(\cdot)}^{\phi}\left(L_{p(\cdot)}\right)\left\|\leq C_{1}\right\|\left(g_{v}\right)_{v \in \mathbb{N}_{0}}\right| \ell_{q(\cdot)}^{\phi}\left(L_{p(\cdot)}\right)\right\|
$$

and

$$
\left\|\left(G_{j}\right)_{j \in \mathbb{N}_{0}}\left|L_{p(\cdot)}^{\phi}\left(\ell_{q(\cdot)}\right)\left\|\leq C_{2}\right\|\left(g_{v}\right)_{v \in \mathbb{N}_{0}}\right| L_{p(\cdot)}^{\phi}\left(\ell_{q(\cdot)}\right)\right\| .
$$

Proof The inequality (2.11) was stated and proved in [12, Lemma 3.5]. Therefore, we are left to prove (2.10).

Let us assume that $p, q \geq 1$. The extension for all $p, q \in \mathcal{P}\left(\mathbb{R}^{n}\right)$ can be done similarly as in Step 2 of the proof of [12, Lemma 3.5] and we omit it here.

Assume that $\left\|\left(g_{v}\right)_{v \in \mathbb{N}_{0}} \mid \ell_{q(\cdot)}^{\phi}\left(L_{p(\cdot)}\right)\right\|=1$, which, by Definition 2.3 and Remark 2.2(iii), implies that, for any cube $P \in \mathcal{Q}$,

$$
\varrho_{\ell_{q(\cdot)}\left(L_{p(\cdot)}\right)}\left(\left(\frac{g_{\nu} \chi_{P}}{\phi(P)}\right)_{\nu \geq\left(j_{P} \vee 0\right)}\right) \leq 1 .
$$

We then fix a cube $P \in \mathcal{Q}$ arbitrarily. Hence,

$$
\begin{aligned}
I(P) & :=\frac{1}{\phi(P)}\left\|\left(G_{j}\right)_{j \geq\left(j_{P} \vee 0\right)} \mid \ell_{q(\cdot)}\left(L_{p(\cdot)}(P)\right)\right\| \\
\leq & \frac{1}{\phi(P)}\left\|\left(\sum_{v=0}^{j} 2^{-(j-v) D_{2}} g_{v} \chi_{P}\right)_{j \geq\left(j_{P} \vee 0\right)} \mid \ell_{q(\cdot)}\left(L_{p(\cdot)}\right)\right\| \\
& +\frac{1}{\phi(P)}\left\|\left(\sum_{\nu=j+1}^{\infty} 2^{-(v-j) D_{1}} g_{\nu} \chi_{P}\right)_{j \geq\left(j_{P} \vee 0\right)} \mid \ell_{q(\cdot)}\left(L_{p(\cdot)}\right)\right\| \\
= & I_{2}(P)+I_{1}(P) .
\end{aligned}
$$

In what follows we will use the notation $c(\varepsilon):=\sum_{l=0}^{\infty} 2^{-l \varepsilon}$, for some $\varepsilon>0$.

Step 1. Firstly we show that there exists some constant $c_{1}>0$ such that $I_{1}(P) \leq c_{1}$. Due to the unit ball property, we turn to the modular and note that 


$$
\begin{aligned}
& \varrho_{\ell_{q(\cdot)}\left(L_{p(\cdot)}\right)}\left(\left(\frac{c_{1}^{-1}}{\phi(P)} \sum_{\nu=j+1}^{\infty} 2^{-(\nu-j) D_{1}} g_{v} \chi_{P}\right)_{j \geq\left(j_{P} \vee 0\right)}\right) \\
& =\sum_{j=\left(j_{P} \vee 0\right)} \inf \left\{\lambda \in(0, \infty):\left\|\frac{c_{1}^{-1}}{\phi(P)} \sum_{\nu=j+1}^{\infty} 2^{-(\nu-j) D_{1}} \frac{g_{\nu} \chi_{P}}{\lambda^{1 / q(\cdot)}} \mid L_{p(\cdot)}\left(\mathbb{R}^{n}\right)\right\| \leq 1\right\} \\
& \leq \sum_{j=\left(j_{P} \vee 0\right)} \inf \left\{\lambda \in(0, \infty): c_{1}^{-1} \sum_{\nu=j+1}^{\infty} 2^{-(\nu-j) D_{1}}\left\|\frac{g_{\nu} \chi_{P}}{\phi(P) \lambda^{1 / q(\cdot)}} \mid L_{p(\cdot)}\left(\mathbb{R}^{n}\right)\right\| \leq 1\right\} .
\end{aligned}
$$

Let us define

$$
I_{1}^{j, \nu}(P):=\inf \left\{\lambda \in(0, \infty): c_{1}^{-1} c(\varepsilon) 2^{-(\nu-j)\left(D_{1}-\varepsilon\right)}\left\|\frac{g_{\nu} \chi_{P}}{\phi(P) \lambda^{1 / q(\cdot)}} \mid L_{p(\cdot)}\left(\mathbb{R}^{n}\right)\right\| \leq 1\right\},
$$

for $j \in \mathbb{N}_{0}, v \geq j+1$ and some $0<\varepsilon<D_{1}$. We claim that, for each $v \geq j+1$, the $\operatorname{sum} \sum_{k=j+1}^{\infty} I_{1}^{j, k}(P)$ is not smaller than the infimum in (2.13). We may assume that this sum is finite. For any $\delta>0$ we have

$$
c_{1}^{-1} c(\varepsilon) 2^{-(\nu-j)\left(D_{1}-\varepsilon\right)}\left\|\frac{g_{\nu} \chi_{P}}{\phi(P)\left[I_{1}^{j, v}(P)+\delta 2^{-v}\right]^{1 / q(\cdot)}} \mid L_{p(\cdot)}\left(\mathbb{R}^{n}\right)\right\| \leq 1,
$$

so that

$$
\begin{aligned}
c_{1}^{-1} c(\varepsilon) & \sum_{\nu=j+1}^{\infty} 2^{-(\nu-j) D_{1}}\left\|\frac{g_{\nu} \chi_{P}}{\phi(P)\left[I_{1}^{j, v}(P)+\delta 2^{-\nu}\right]^{1 / q(\cdot)}} \mid L_{p(\cdot)}\left(\mathbb{R}^{n}\right)\right\| \\
\leq & \sum_{\nu=j+1}^{\infty} 2^{-(\nu-j) \varepsilon} \leq \sum_{l=0}^{\infty} 2^{-l \varepsilon} .
\end{aligned}
$$

Therefore

$$
c_{1}^{-1} \sum_{\nu=j+1}^{\infty} 2^{-(\nu-j) D_{1}}\left\|\frac{g_{\nu} \chi_{P}}{\phi(P) \sum_{k=j+1}^{\infty}\left[I_{1}^{j, k}(P)+\delta 2^{-k}\right]^{1 / q(\cdot)}} \mid L_{p(\cdot)}\left(\mathbb{R}^{n}\right)\right\| \leq 1
$$

and so

$$
\begin{aligned}
& \inf \left\{\lambda \in(0, \infty): c_{1}^{-1} \sum_{\nu=j+1}^{\infty} 2^{-(\nu-j) D_{1}}\left\|\frac{g_{\nu} \chi_{P}}{\phi(P) \lambda^{1 / q(\cdot)}} \mid L_{p(\cdot)}\left(\mathbb{R}^{n}\right)\right\| \leq 1\right\} \\
& \quad \leq \sum_{k=j+1}^{\infty}\left(I_{1}^{j, k}(P)+\delta 2^{-k}\right) .
\end{aligned}
$$


The claim follows then by the convergence of the second part of the series on the right-hand side and the arbitrariness of $\delta>0$. Now using this in (2.13), we have

$$
\begin{aligned}
& \varrho_{\ell_{q(\cdot)}\left(L_{p(\cdot)}\right)}\left(\left(\frac{c_{1}^{-1}}{\phi(P)} \sum_{\nu=j+1}^{\infty} 2^{-(k-j) D_{1}} g_{k} \chi_{P}\right)_{j \geq\left(j_{P} \vee 0\right)}\right) \\
& \leq \sum_{j=\left(j_{P} \vee 0\right)}^{\infty} \sum_{k=j+1}^{\infty} \inf \left\{\lambda \in(0, \infty): c_{1}^{-1} c(\varepsilon) 2^{-(k-j)\left(D_{1}-\varepsilon\right)}\left\|\frac{g_{k} \chi_{P}}{\phi(P) \lambda^{1 / q(\cdot)}} \mid L_{p(\cdot)}\left(\mathbb{R}^{n}\right)\right\| \leq 1\right\} \\
& \leq c_{1}^{-1} c(\varepsilon) \sum_{k=\left(j_{P} \vee 0\right)}^{\infty} \sum_{j=\left(j_{P} \vee 0\right)}^{k} 2^{-(k-j)\left(D_{1}-\varepsilon\right)} . \\
& \quad \cdot \inf \left\{\lambda \in(0, \infty):\left\|\frac{g_{k} \chi_{P}}{\phi(P) \lambda^{1 / q(\cdot)}} \mid L_{p(\cdot)}\left(\mathbb{R}^{n}\right)\right\| \leq 1\right\} \\
& \leq \sum_{k=\left(j_{P} \vee 0\right)}^{\infty} \inf \left\{\lambda \in(0, \infty):\left\|\frac{g_{k} \chi_{P}}{\phi(P) \lambda^{1 / q(\cdot)}} \mid L_{p(\cdot)}\left(\mathbb{R}^{n}\right)\right\| \leq 1\right\} \\
& \leq 1,
\end{aligned}
$$

doing a convenient change of variables and with the choice of $c_{1}=c(\varepsilon) c\left(D_{1}-\varepsilon\right)$. The first part is then proved.

Step 2. We prove now that $I_{2}(P) \leq c_{2}$, for some $c_{2}>0$. We proceed similarly as before, and get, for $0<\varepsilon<\min \left\{D_{2}, D_{2}-\log _{2} \tilde{c}_{1}(\phi)\right\}$,

$$
\begin{aligned}
& \varrho_{\ell_{q(\cdot)}\left(L_{p(\cdot)}\right)}\left(\left(\frac{c_{2}^{-1}}{\phi(P)} \sum_{v=0}^{j} 2^{-(j-k) D_{2}} g_{k} \chi_{P}\right)_{j \geq\left(j_{P} \vee 0\right)}\right) \\
& \leq \sum_{j=\left(j_{P} \vee 0\right)}^{\infty} \sum_{k=0}^{j} \inf \left\{\lambda \in(0, \infty): c_{2}^{-1} c(\varepsilon) 2^{-(j-k)\left(D_{2}-\varepsilon\right)}\left\|\frac{g_{k} \chi_{P}}{\phi(P) \lambda^{1 / q(\cdot)}} \mid L_{p(\cdot)}\left(\mathbb{R}^{n}\right)\right\| \leq 1\right\} \\
& \leq \sum_{k=0}^{\infty} \sum_{j=\left(j_{P} \vee 0 \vee k\right)}^{\infty} c_{2}^{-1} c(\varepsilon) 2^{-(j-k)\left(D_{2}-\varepsilon\right)} \text {. } \\
& \cdot \inf \left\{\lambda \in(0, \infty):\left\|\frac{g_{k} \chi_{P}}{\phi(P) \lambda^{1 / q(\cdot)}} \mid L_{p(\cdot)}\left(\mathbb{R}^{n}\right)\right\| \leq 1\right\} \\
& =\sum_{k=0}^{\left(j_{P} \vee 0\right)-1} \sum_{j=\left(j_{P} \vee 0\right)}^{\infty} \cdots+\sum_{k=\left(j_{P} \vee 0\right)}^{\infty} \sum_{j=k}^{\infty} \cdots \\
& =: H_{1}(P)+H_{2}(P)
\end{aligned}
$$

for the same $c(\varepsilon)$ as before. For $H_{2}(P)$, after a proper change of variables, and choosing $c_{2} \geq \bar{c}:=2 c(\varepsilon) c\left(D_{2}-\varepsilon\right)$, we have 


$$
\begin{aligned}
H_{2}(P)= & c_{2}^{-1} c(\varepsilon) \sum_{k=\left(j_{P} \vee 0\right)}^{\infty} \sum_{j=k}^{\infty} 2^{-(j-k)\left(D_{2}-\varepsilon\right) .} \\
& \cdot \inf \left\{\lambda \in(0, \infty): \| \frac{g_{k} \chi_{P}}{\left.\phi(P) \lambda^{1 / q(\cdot)} \mid L_{p(\cdot)}\left(\mathbb{R}^{n}\right) \| \leq 1\right\}}\right. \\
= & c_{2}^{-1} c(\varepsilon) c\left(D_{2}-\varepsilon\right) . \\
& \cdot \sum_{k=\left(j_{P} \vee 0\right)}^{\infty} \inf \left\{\lambda \in(0, \infty):\left\|\frac{g_{k} \chi_{P}}{\phi(P) \lambda^{1 / q(\cdot)}} \mid L_{p(\cdot)}\left(\mathbb{R}^{n}\right)\right\| \leq 1\right\} \\
\leq & \frac{1}{2} \varrho_{\ell_{q(\cdot)}\left(L_{p(\cdot)}\right)}\left(\left(\frac{g_{k} \chi_{P}}{\phi(P)}\right)_{k \geq\left(j_{P} \vee 0\right)}\right) \\
\leq & \frac{1}{2} .
\end{aligned}
$$

As for $H_{1}(P)$, we proceed similarly. We then get

$$
\begin{aligned}
H_{1}(P)= & c_{2}^{-1} c(\varepsilon) \sum_{k=0}^{\left(j_{P} \vee 0\right)-1} \sum_{j=\left(j_{P} \vee 0\right)}^{\infty} 2^{-(j-k)\left(D_{2}-\varepsilon\right)} . \\
& \cdot \inf \left\{\lambda \in(0, \infty):\left\|\frac{g_{k} \chi_{P}}{\phi(P) \lambda^{1 / q(\cdot)}} \mid L_{p(\cdot)}\left(\mathbb{R}^{n}\right)\right\| \leq 1\right\} \\
= & c_{2}^{-1} c(\varepsilon) \sum_{k=0}^{\left(j_{P} \vee 0\right)-1} 2^{k\left(D_{2}-\varepsilon\right)} \sum_{j=\left(j_{P} \vee 0\right)}^{\infty} 2^{-j\left(D_{2}-\varepsilon\right)} . \\
& \cdot \inf \left\{\lambda \in(0, \infty):\left\|\frac{g_{k} \chi_{P}}{\phi(P) \lambda^{1 / q(\cdot)}} \mid L_{p(\cdot)}\left(\mathbb{R}^{n}\right)\right\| \leq 1\right\} \\
= & \frac{c_{2}^{-1} c(\varepsilon)}{1-2^{-\left(D_{2}-\varepsilon\right)}} \sum_{k=0}^{\left(j_{P} \vee 0\right)-1} 2^{-\left(\left(j_{P} \vee 0\right)-k\right)\left(D_{2}-\varepsilon\right)} . \\
& \cdot \frac{1}{\phi(P)} \inf \left\{\lambda \in(0, \infty):\left\|\frac{g_{k} \chi_{P}}{\lambda^{1 / q(\cdot)}} \mid L_{p(\cdot)}\left(\mathbb{R}^{n}\right)\right\| \leq 1\right\} \\
\leq & \frac{c_{2}^{-1} c(\varepsilon)}{1-2^{-\left(D_{2}-\varepsilon\right)}} \sum_{k=0}^{\left(j_{P} \vee 0\right)-1} 2^{-\left(\left(j_{P} \vee 0\right)-k\right)\left(D_{2}-\varepsilon-\log _{2} \tilde{c}_{1}(\phi)\right)} \cdot \\
& \cdot \varrho_{\ell_{q(\cdot)}\left(L_{p(\cdot)}\right)}\left(\left(\frac{g_{k} \chi_{R}}{\phi(R)}\right)_{k \geq\left(j_{R} \vee 0\right)}\right) \\
&
\end{aligned}
$$

with $R=Q\left(c_{P}, 2^{\left(\mathrm{J}_{P} \vee 0\right)-k-j_{P}}\right)$ and choosing

$$
c_{2} \geq \tilde{c}:=\frac{2 c(\varepsilon) c\left(D_{2}-\varepsilon-\log _{2} \tilde{c}_{1}(\phi)\right)}{1-2^{-\left(D_{2}-\varepsilon\right)}} .
$$


In the fourth step we have used (2.5) and the fact that

$$
\begin{aligned}
& \inf \left\{\lambda \in(0, \infty):\left\|\frac{g_{k} \chi_{P}}{\lambda^{1 / q(\cdot)}} \mid L_{p(\cdot)}\left(\mathbb{R}^{n}\right)\right\| \leq 1\right\} \\
& \quad \leq \inf \left\{\lambda \in(0, \infty):\left\|\frac{g_{k} \chi_{R}}{\lambda^{1 / q(\cdot)}} \mid L_{p(\cdot)}\left(\mathbb{R}^{n}\right)\right\| \leq 1\right\} .
\end{aligned}
$$

We then obtain $I_{2}(P) \leq c_{2}$ by considering $c_{2} \geq \max \{\bar{c}, \tilde{c}\}$, as we wanted to prove.

Remark 2.13 Naturally, this statement holds also true if the indices $j$ and $v$ run only over natural numbers.

\subsection{Variable 2-microlocal Besov-type and Triebel-Lizorkin-type spaces}

We will present now the definition of the spaces under consideration in this paper. To do this, we start by introducing the notions of admissible weight sequence and admissible pair of functions.

Definition 2.14 Let $\alpha \geq 0$ and $\alpha_{1}, \alpha_{2} \in \mathbb{R}$ with $\alpha_{1} \leq \alpha_{2}$. A sequence of non-negative measurable functions in $\mathbb{R}^{n} \boldsymbol{w}=\left(w_{j}\right)_{j \in \mathbb{N}_{0}}$ belongs to the class $\mathcal{W}_{\alpha_{1}, \alpha_{2}}^{\alpha}\left(\mathbb{R}^{n}\right)$ if the following conditions are satisfied:

(i) There exists a constant $c>0$ such that

$$
0<w_{j}(x) \leq c w_{j}(y)\left(1+2^{j}|x-y|\right)^{\alpha} \quad \text { for all } j \in \mathbb{N}_{0} \text { and all } x, y \in \mathbb{R}^{n} .
$$

(ii) For all $j \in \mathbb{N}_{0}$ it holds

$$
2^{\alpha_{1}} w_{j}(x) \leq w_{j+1}(x) \leq 2^{\alpha_{2}} w_{j}(x) \quad \text { for all } x \in \mathbb{R}^{n} .
$$

Such a system $\left(w_{j}\right)_{j \in \mathbb{N}_{0}} \in \mathcal{W}_{\alpha_{1}, \alpha_{2}}^{\alpha}\left(\mathbb{R}^{n}\right)$ is called admissible weight sequence.

Properties of admissible weights may be found in [15, Remark 2.4].

Definition 2.15 We say that a pair $\left(\varphi, \varphi_{0}\right)$ of functions in $\mathscr{S}\left(\mathbb{R}^{n}\right)$ is admissible if

$$
\operatorname{supp} \hat{\varphi} \subset\left\{\xi \in \mathbb{R}^{n}: \frac{1}{2} \leq|\xi| \leq 2\right\} \text { and }|\widehat{\varphi}(\xi)|>0 \text { when } \frac{3}{5} \leq|\xi| \leq \frac{5}{3}
$$

and

$$
\text { supp } \hat{\varphi}_{0} \subset\left\{\xi \in \mathbb{R}^{n}:|\xi| \leq 2\right\} \text { and }\left|\hat{\varphi}_{0}(\xi)\right|>0 \text { when }|\xi| \leq \frac{5}{3} \text {. }
$$

Further, we set $\varphi_{j}(x):=2^{j n} \varphi\left(2^{j} x\right)$ for $j \in \mathbb{N}$. Then $\left(\varphi_{j}\right)_{j \in \mathbb{N}_{0}} \subset \mathscr{S}\left(\mathbb{R}^{n}\right)$ and

$$
\operatorname{supp} \varphi_{j} \subset\left\{x \in \mathbb{R}^{n}: 2^{j-1} \leq|x| \leq 2^{j+1}\right\} .
$$


We are finally in a position to introduce variable 2-microlocal Besov-type and Triebel-Lizorkin-type spaces.

Definition 2.16 Let $\left(\varphi, \varphi_{0}\right)$ be a pair of admissible functions on $\mathbb{R}^{n}$. Let $p, q \in \mathcal{P}^{\log }\left(\mathbb{R}^{n}\right), \boldsymbol{w}=\left(w_{j}\right)_{j \in \mathbb{N}_{0}} \in \mathcal{W}_{\alpha_{1}, \alpha_{2}}^{\alpha}\left(\mathbb{R}^{n}\right)$ and $\phi \in \mathcal{G}\left(\mathbb{R}_{+}^{n+1}\right)$.

(i) The variable 2-microlocal Besov-type space $B_{p(\cdot), q(\cdot)}^{w, \phi}\left(\mathbb{R}^{n}\right)$ is defined to be the set of all $f \in \mathscr{S}\left(\mathbb{R}^{n}\right)$ such that

$$
\left\|f\left|B_{p(\cdot), q(\cdot)}^{w, \phi}\left(\mathbb{R}^{n}\right)\|:=\|\left(w_{j}(\cdot)\left(\varphi_{j} * f\right)(\cdot)\right)_{j \in \mathbb{N}_{0}}\right| \ell_{q(\cdot)}^{\phi}\left(L_{p(\cdot)}\right)\right\|<\infty .
$$

(ii) Assume $p^{+}, q^{+}<\infty$. The variable 2-microlocal Triebel-Lizorkin-type space $F_{p(\cdot), q(\cdot)}^{w, \phi}\left(\mathbb{R}^{n}\right)$ is defined to be the set of all $f \in \mathscr{S}\left(\mathbb{R}^{n}\right)$ such that

$$
\left\|f\left|F_{p(\cdot), q(\cdot)}^{\boldsymbol{w}, \phi}\left(\mathbb{R}^{n}\right)\|:=\|\left(w_{j}(\cdot)\left(\varphi_{j} * f\right)(\cdot)\right)_{j \in \mathbb{N}_{0}}\right| L_{p(\cdot)}^{\phi}\left(\ell_{q(\cdot)}\right)\right\|<\infty .
$$

\section{Remark 2.17}

(i) These spaces were introduced by Wu et al. in [37], where the authors have proved the independence of the spaces on the admissible pair.

(ii) In the particular case of $w_{j}(\cdot)=2^{j s(\cdot)}$, with $s \in C_{\operatorname{loc}}^{\log }\left(\mathbb{R}^{n}\right)$, we recover $A_{p(\cdot), q(\cdot)}^{s(\cdot),}\left(\mathbb{R}^{n}\right), A \in\{B, F\}$, introduced in [38, 39] and also investigated in [12].

(iii) When $\phi \equiv 1$, then $A_{p(\cdot), q(\cdot)}^{w, \phi}\left(\mathbb{R}^{n}\right)=A_{p(\cdot), q(\cdot)}^{w}\left(\mathbb{R}^{n}\right), A \in\{B, F\}$, are the 2-microlocal Besov and Triebel-Lizorkin spaces with variable exponents. For a good overview on this scale we recommend $[1,2]$.

(iv) When $p(\cdot)=p, q(\cdot)=q$ are constant exponents, $w_{j}(\cdot)=2^{j s}, j \in \mathbb{N}_{0}$, and $\phi(Q):=|Q|^{\tau}$ for all cubes $Q$ and $\tau \in[0, \infty)$, then $A_{p(\cdot), q(\cdot)}^{w, \phi}\left(\mathbb{R}^{n}\right)=A_{p, q}^{s, \tau}\left(\mathbb{R}^{n}\right)$, $A \in\{B, F\}$, are the Besov-type and Triebel-Lizorkin-type spaces introduced by Yuan et al. in [40].

\section{Maximal functions and local means characterization}

Let $\left(\psi_{j}\right)_{j \in \mathbb{N}_{0}}$ be a sequence in $\mathscr{S}\left(\mathbb{R}^{n}\right)$. For each $f \in \mathscr{S}\left(\mathbb{R}^{n}\right)$ and $a>0$, the Peetre's maximal functions were defined by Peetre [28] by

$$
\left(\psi_{j}^{*} f\right)_{a}(x):=\sup _{y \in \mathbb{R}^{n}} \frac{\left|\psi_{j} * f(y)\right|}{\left(1+\left|2^{j}(x-y)\right|\right)^{a}}, \quad x \in \mathbb{R}^{n}, j \in \mathbb{N}_{0} .
$$

In [37, Theorems 4.5 and 4.7], the authors proved a characterization of $B_{p(\cdot), q(\cdot)}^{w, \phi}\left(\mathbb{R}^{n}\right)$ and $F_{p(\cdot), q(\cdot)}^{w, \phi}\left(\mathbb{R}^{n}\right)$ using the Peetre's maximal functions, but where the sequence $\left(\psi_{j}\right)_{j \in \mathbb{N}_{0}}$ is the same as in Definition 2.16, which is built upon an admissible pair. Here we intend to extend those results, by showing that they still hold if one considers more general pairs of functions. Additionally, we also prove that one can replace 
the admissible pairs in the definition of $B_{p(\cdot), q(\cdot)}^{w, \phi}\left(\mathbb{R}^{n}\right)$ and $F_{p(\cdot), q(\cdot)}^{w, \phi}\left(\mathbb{R}^{n}\right)$ by more general ones (in terms of equivalent quasi-norms). The main result of this section reads then as follows.

Theorem 3.1 Let $p, q \in \mathcal{P}^{\log }\left(\mathbb{R}^{n}\right), \boldsymbol{w}=\left(w_{j}\right)_{j \in \mathbb{N}_{0}} \in \mathcal{W}_{\alpha_{1}, \alpha_{2}}^{\alpha}\left(\mathbb{R}^{n}\right)$ and $\phi \in \mathcal{G}\left(\mathbb{R}_{+}^{n+1}\right)$. Let $R \in \mathbb{N}_{0}$ with $R>\alpha_{2}+\max \left\{0, \log _{2} \tilde{c_{1}}(\phi)\right\}$, where $\tilde{c_{1}}(\phi)$ is the constant in (2.5), and let $\psi_{0}, \psi \in \mathscr{S}\left(\mathbb{R}^{n}\right)$ be such that

$$
\left(D^{\beta} \widehat{\psi}\right)(0)=0 \quad \text { for } \quad 0 \leq|\beta|<R
$$

and

$$
\begin{gathered}
\left|\widehat{\psi}_{0}(\xi)\right|>0 \quad \text { on } \quad\left\{\xi \in \mathbb{R}^{n}:|\xi| \leq k \varepsilon\right\}, \\
|\widehat{\psi}(\xi)|>0 \quad \text { on } \quad\left\{\xi \in \mathbb{R}^{n}: \frac{\varepsilon}{2} \leq|\xi| \leq k \varepsilon\right\},
\end{gathered}
$$

for some $\varepsilon>0$ and $k \in] 1,2]$.

(i) For

$$
a>\frac{n}{p^{-}}+c_{\log }\left(\frac{1}{q}\right)+\alpha+\max \left\{0, \log _{2} \tilde{c_{1}}(\phi)\right\},
$$

we have

$$
\begin{aligned}
& \left\|f\left|B_{p(\cdot), q(\cdot)}^{w, \phi}\left(\mathbb{R}^{n}\right)\|\sim\|\left(w_{j}(\cdot)\left(\psi_{j}^{*} f\right)_{a}\right)_{j \in \mathbb{N}_{0}}\right| \ell_{q(\cdot)}^{\phi}\left(L_{p(\cdot)}\right)\right\| \sim\left\|\left(w_{j}(\cdot)\left(\psi_{j} * f\right)\right)_{j \in \mathbb{N}_{0}} \mid \ell_{q(\cdot)}^{\phi}\left(L_{p(\cdot)}\right)\right\| \\
& \quad \text { for all } f \in \mathscr{S}\left(\mathbb{R}^{n}\right) . \\
& \text { (ii) Assume } p^{+}, q^{+}<\infty \text {. For }
\end{aligned}
$$

$a>\frac{n}{\min \left\{p^{-}, q^{-}\right\}}+\alpha+\max \left\{0, \log _{2} \tilde{c_{1}}(\phi)\right\}$,

we have

$$
\begin{aligned}
\left\|f \mid F_{p(\cdot), q(\cdot)}^{w, \phi}\left(\mathbb{R}^{n}\right)\right\| & \sim\left\|\left(w_{j}(\cdot)\left(\psi_{j}^{*} f\right)_{a}\right)_{j \in \mathbb{N}_{0}}\left|L_{p(\cdot)}^{\phi}\left(\ell_{q(\cdot)}\right)\|\sim\|\left(w_{j}(\cdot)\left(\psi_{j} * f\right)\right)_{j \in \mathbb{N}_{0}}\right| L_{p(\cdot)}^{\phi}\left(\ell_{q(\cdot)}\right)\right\| \\
\text { for all } f & \in \mathscr{S}\left(\mathbb{R}^{n}\right) .
\end{aligned}
$$

\section{Remark 3.2}

(i) The conditions (3.1) are usually called moment conditions, while (3.2) and (3.3) are the so-called Tauberian conditions. If $R=0$ then no moment conditions (3.1) on $\psi$ are required. 
(ii) The case $\phi \equiv 1$ is covered by [1, Theorem 3.1(ii)], where we can find also a discussion on the importance of having a $k$ in the conditions (3.2) and (3.3) in contrast with the case $k=2$ usually found in the literature in such type of result, cf. e.g. [16, 19, 29].

(iii) When $p(\cdot)=p, q(\cdot)=q$ are constant exponents, $\phi(Q):=|Q|^{\tau}$ for all cubes $Q$ and $\tau \in[0, \infty)$, and $w_{j}(x)=2^{j s}$ for all $x \in \mathbb{R}^{n}, j \in \mathbb{N}_{0}$ and $s \in \mathbb{R}$, then $\log _{2} \tilde{c_{1}}(\phi)=n \tau$ and such a characterization with $k=2$ has been already established in the homogeneous case by Yang and Yuan, cf. [41, Theorem 2.1(ii)].

(iv) In [37] the authors proved the independence of the spaces from the admissible pair as a consequence of the $\varphi$-transform characterization. The above theorem provides an alternative proof, since an admissible pair satisfies conditions (3.2) and (3.3) with $\varepsilon=\frac{6}{5}$ and $k=\frac{25}{18}$. Moreover, it becomes clear that $B_{p(\cdot), q(\cdot)}^{w, \phi}\left(\mathbb{R}^{n}\right)$ and $F_{p(\cdot), q(\cdot)}^{w, \phi}\left(\mathbb{R}^{n}\right)$ can be defined using more general pairs than the admissible ones (in the sense of equivalent quasi-norms). We refer in particular to the case stated in Corollary 3.4 below.

Remark 3.3 The proof of Theorem 3.1 can be carried out following the proof done by Rychkov [29] in the classical case. Part (ii) is an extension of [12, Theorem 3.2], where the spaces $F_{p(\cdot), q(\cdot)}^{s(\cdot) \phi}\left(\mathbb{R}^{n}\right)$ were considered. As noticed in [12, Remark 3.9], the proof can easily be adapted for the more general scale $F_{p(\cdot), q(\cdot)}^{w, \phi}\left(\mathbb{R}^{n}\right)$. In a similar way, one can prove the result for the variable 2-microlocal Besov-type spaces $B_{p(\cdot), q(\cdot)}^{w, \phi}\left(\mathbb{R}^{n}\right)$. In this case, the discrete convolution inequality stated in Lemma 2.10(i) is of great importance.

Lastly in this section we present an important application of Theorem 3.1, that is when $\psi_{0}$ and $\psi$, satisfying (3.1)-(3.3), are local means. The name comes from the compact support of $\psi_{0}:=k_{0}$ and $\psi:=k$, which is admitted in the following statement.

Corollary 3.4 Let $p, q \in \mathcal{P}^{\log }\left(\mathbb{R}^{n}\right) \quad$ (with $p^{+}, q^{+}<\infty \quad$ in the F-case), $\boldsymbol{w}=\left(w_{j}\right)_{j \in \mathbb{N}_{0}} \in \mathcal{W}_{\alpha_{1}, \alpha_{2}}^{\alpha}\left(\mathbb{R}^{n}\right)$ and $\phi \in \mathcal{G}\left(\mathbb{R}_{+}^{n+1}\right)$. For given $N \in \mathbb{N}_{0}$ and $d>0$, let $k_{0}, k \in \mathscr{S}\left(\mathbb{R}^{n}\right)$ with supp $k_{0}$, supp $k \subset d Q_{0,0}$,

$$
\left(D^{\beta} \widehat{k}\right)(0)=0 \quad \text { if } \quad 0 \leq|\beta|<N,
$$

$\widehat{k_{0}}(0) \neq 0$ and $\hat{k}(x) \neq 0$ if $0<|x|<\varepsilon$, for some $\varepsilon>0$. If $N>\alpha_{2}+\max \left\{0, \log _{2} \tilde{c_{1}}(\phi)\right\}$, then

$$
\left\|f\left|B_{p(\cdot), q(\cdot)}^{w, \phi}\left(\mathbb{R}^{n}\right)\|\sim\|\left(w_{j}(\cdot)\left(k_{j} * f\right)\right)_{j \in \mathbb{N}_{0}}\right| \ell_{q(\cdot)}^{\phi}\left(L_{p(\cdot)}\right)\right\|
$$

and

$$
\left\|f\left|F_{p(\cdot), q(\cdot)}^{\boldsymbol{w}, \phi}\left(\mathbb{R}^{n}\right)\|\sim\|\left(w_{j}(\cdot)\left(k_{j} * f\right)\right)_{j \in \mathbb{N}_{0}}\right| L_{p(\cdot)}^{\phi}\left(\ell_{q(\cdot)}\right)\right\|
$$

for all $f \in \mathscr{S}\left(\mathbb{R}^{n}\right)$. 
Proof It is clear the existence of $k_{0}, k^{0} \in \mathscr{S}\left(\mathbb{R}^{n}\right)$ with supp $k_{0}$, supp $k^{0} \subset d Q_{0,0}$, $\widehat{k_{0}}(0) \neq 0$ and $\widehat{k^{0}}(0) \neq 0$. Then, following $[32,11.2]$ and taking $M \in \mathbb{N}_{0}$ with $2 M \geq N$ define $k:=\Delta^{M} k^{0}$. Since $\hat{k}(x)=\left(-\sum_{i=1}^{n}\left|x_{i}\right|^{2}\right)^{M} \hat{k^{0}}(x)$, we immediately have (3.4) and $\hat{k}(x) \neq 0$ if $0<|x|<\varepsilon$, for a small enough $\varepsilon>0$. The rest is a direct consequence of Theorem 3.1.

\section{Non-smooth atomic decomposition}

In [37] the authors obtained a characterization of the spaces $B_{p(\cdot), q(\cdot)}^{w, \phi}\left(\mathbb{R}^{n}\right)$ and $F_{p(\cdot), q(\cdot)}^{w, \phi}\left(\mathbb{R}^{n}\right)$ by smooth atomic decompositions, generalizing previous results obtained in [8, 17, 38-40] for the particular cases described in Remark 2.17. We recall the result from [37] and start by defining smooth atoms and appropriate sequence spaces, where we opted here for a different normalization.

Definition 4.1 Let $K, L \in \mathbb{N}_{0}$. A function $a_{Q} \in C^{K}\left(\mathbb{R}^{n}\right)$ is called a $[K, L]$-smooth atom centered at $Q:=Q_{v k} \in \mathcal{Q}$, where $v \in \mathbb{N}_{0}$ and $k \in \mathbb{Z}^{n}$, if

$$
\operatorname{supp} a_{Q} \subset 3 Q,\left\|a_{Q}\left(2^{-\nu} \cdot\right) \mid C^{K}\left(\mathbb{R}^{n}\right)\right\| \leq 1,
$$

and, when $v \in \mathbb{N}$,

$$
\int_{\mathbb{R}^{n}} x^{\gamma} a_{Q}(x) d x=0
$$

for all multi-indices $\gamma \in \mathbb{N}_{0}^{n}$ with $|\gamma|<L$.

Remark 4.2 As usual when $L=0$ no moment conditions are required by (4.2).

Definition 4.3 Let $p, q \in \mathcal{P}^{\log }\left(\mathbb{R}^{n}\right), \boldsymbol{w}=\left(w_{\nu}\right)_{\nu \in \mathbb{N}_{0}} \in \mathcal{W}_{\alpha_{1}, \alpha_{2}}^{\alpha}\left(\mathbb{R}^{n}\right)$ and $\phi \in \mathcal{G}\left(\mathbb{R}_{+}^{n+1}\right)$.

(i) The sequence space $b_{p(\cdot), q(\cdot)}^{w, \phi}\left(\mathbb{R}^{n}\right)$ is defined as the set of all sequences $t:=\left\{t_{Q}\right\}_{Q \in \mathcal{Q}^{*}} \subset \mathbb{C}$ such that

$$
\left\|t\left|b_{p(\cdot), q(\cdot)}^{w, \phi}\left(\mathbb{R}^{n}\right)\|:=\|\left(\sum_{m \in \mathbb{Z}^{n}} w_{v}\left(2^{-v} k\right)\left|t_{Q_{v m}}\right| \chi_{Q_{v m}}\right)_{v \in \mathbb{N}_{0}}\right| \ell_{q(\cdot)}^{\phi}\left(L_{p(\cdot)}\right)\right\|<\infty .
$$

(ii) Assume $p^{+}, q^{+}<\infty$. The sequence space $f_{p(\cdot), q(\cdot)}^{w, \phi}\left(\mathbb{R}^{n}\right)$ is defined as the set of all sequences $t:=\left\{t_{Q}\right\}_{Q \in \mathcal{Q}^{*}} \subset \mathbb{C}$ such that

$$
\left\|t\left|f_{p(\cdot), q(\cdot)}^{w, \phi}\left(\mathbb{R}^{n}\right)\|:=\|\left(\sum_{m \in \mathbb{Z}^{n}} w_{v}\left(2^{-v} k\right)\left|t_{Q_{v m}}\right| \chi_{Q_{v m}}\right)_{v \in \mathbb{N}_{0}}\right| L_{p(\cdot)}^{\phi}\left(\ell_{q(\cdot)}\right)\right\|<\infty .
$$


Next we present the notion of non-smooth atoms already used in [10] in the context of 2-microlocal spaces with variable exponents and which were slightly adapted from [30]. Note that the usual parameters $K$ and $L$ are now non-negative real numbers instead of non-negative integer numbers.

Definition 4.4 Let $K, L \geq 0$. A function $a_{Q}: \mathbb{R}^{n} \rightarrow \mathbb{C}$ is called a $[K, L]$-non-smooth atom centered at $Q:=Q_{v k} \in \mathcal{Q}$, with $\nu \in \mathbb{N}_{0}$ and $k \in \mathbb{Z}^{n}$, if

$$
\begin{gathered}
\operatorname{supp} a_{Q} \subset 3 Q, \\
\left\|a_{Q}\left(2^{-\nu} \cdot\right) \mid \mathscr{C}^{K}\left(\mathbb{R}^{n}\right)\right\| \leq 1,
\end{gathered}
$$

and for every $\psi \in \mathscr{C}^{L}\left(\mathbb{R}^{n}\right)$ it holds

$$
\left|\int_{\mathbb{R}^{n}} \psi(x) a_{Q}(x) d x\right| \leq c 2^{-v(L+n)}\left\|\psi \mid \mathscr{C}^{L}\left(\mathbb{R}^{n}\right)\right\| .
$$

Remark 4.5 Since $C^{k}\left(\mathbb{R}^{n}\right) \hookrightarrow \mathscr{C}^{k}\left(\mathbb{R}^{n}\right)$ for $k \in \mathbb{N}_{0}$, it is clear that condition (4.4) follows from (4.1). Moreover, using a Taylor expansion, (4.5) can be derived from (4.2) when $L \in \mathbb{N}$, cf. [30, Remark 3.4]. Therefore, when $K, L \in \mathbb{N}_{0}$, any $[K, L]$-smooth atom is a $[K, L]$-non-smooth atom. Moreover, both conditions (4.4) and (4.5) are ordered in $K$ and $L$, i.e. the conditions are stricter for increasing $K$ and $L$, see [30, Remark. 3.4].

For the next two auxiliary results we refer to [10, Lemmas 3.6,3.7].

Lemma 4.6 Let $k_{j}$ be the local means according to Corollary3.4 with $d=3$. Then $c 2^{-j n} k_{j}$ is a $[K, L]$-non-smooth atom centered at $Q_{j 0}$, for some constant $c>0$ independently of $j$ and for arbitrary large $K>0$ and $L \leq N+1$.

Lemma 4.7 Let $k_{j}$ be the local means according to Corollary 3.4 with $d=3$. Let also $\left(a_{Q}\right)_{Q \in \mathcal{Q}^{*}}$ be $[K, L]$-non-smooth atoms. Then, with $Q=Q_{v, k}, v \in \mathbb{N}_{0}, k \in \mathbb{Z}^{n}$, it holds

$$
\left|\int_{\mathbb{R}^{n}} k_{j}(y) a_{Q}(x-y) d y\right| \leq c 2^{-(j-v) K} \chi(c Q)(x), \quad \text { for } j \geq v
$$

and

$$
\left|\int_{\mathbb{R}^{n}} k_{j}(x-y) a_{Q}(y) d y\right| \leq c 2^{-(\nu-j)(L+n)} \chi\left(c 2^{v-j} Q\right)(x), \quad \text { for } j<v .
$$

We are now ready to state the main theorems of this section. We start by presenting the result for the spaces $F_{p(\cdot), q(\cdot)}^{w, \phi}\left(\mathbb{R}^{n}\right)$. However, we won't present the proof here, as it can be carried out in the same way as the proof of [12, Theorem 4.10]. Due to the use of an admissible weight sequence $w \in \mathcal{W}_{\alpha_{1}, \alpha_{2}}^{\alpha}\left(\mathbb{R}^{n}\right)$ in our case, we only have to do some minor adjustments. 
Theorem 4.8 Let $p, q \in \mathcal{P}^{\log }\left(\mathbb{R}^{n}\right)$ with $p^{+}, q^{+}<\infty, \boldsymbol{w}=\left(w_{j}\right)_{j \in \mathbb{N}_{0}} \in \mathcal{W}_{\alpha_{1}, \alpha_{2}}^{\alpha}\left(\mathbb{R}^{n}\right)$ and $\phi \in \mathcal{G}\left(\mathbb{R}_{+}^{n+1}\right)$.

(i) Let $K, L \geq 0$ with

$$
K>\alpha_{2}+\max \left\{0, \log _{2} \tilde{c}_{1}(\phi)\right\} \quad \text { and } \quad L>\frac{n}{\min \left\{1, p^{-}, q^{-}\right\}}-n-\alpha_{1} .
$$

Suppose that $\left\{a_{Q}\right\}_{Q \in \mathcal{Q}^{*}}$ is a family of $[K, L]$-non-smooth atoms and that $\left\{t_{Q}\right\}_{Q \in \mathcal{Q}^{*}} \in f_{p(\cdot), q(\cdot)}^{w, \phi}\left(\mathbb{R}^{n}\right)$. Then $f:=\sum_{Q \in \mathcal{Q}^{*}} t_{Q} a_{Q}$ converges in $\mathscr{S}\left(\mathbb{R}^{n}\right)$ and

$$
\left\|f\left|F_{p(\cdot), q(\cdot)}^{\boldsymbol{w}, \phi}\left(\mathbb{R}^{n}\right)\|\leq c\| t\right| f_{p(\cdot), q(\cdot)}^{\boldsymbol{w}, \phi}\left(\mathbb{R}^{n}\right)\right\|
$$

with $c$ being a positive constant independent of $t$.

(ii) Conversely, if $f \in F_{p(\cdot), q(\cdot)}^{w, \phi}\left(\mathbb{R}^{n}\right)$, then, for any given $K, L \geq 0$, there exist a sequence $\left\{t_{Q}\right\}_{Q \in \mathcal{Q}^{*}} \in f_{p(\cdot), q(\cdot)}^{w, \phi}\left(\mathbb{R}^{n}\right)$ and a sequence $\left\{a_{Q}\right\}_{Q \in \mathcal{Q}^{*}}$ of $[K, L]-n o n-$ smooth atoms such that $f=\sum_{Q \in \mathcal{Q}^{*}} t_{Q} a_{Q}$ in $\mathscr{S}\left(\mathbb{R}^{n}\right)$ and

$$
\left\|t\left|f_{p(\cdot), q(\cdot)}^{\boldsymbol{w}, \phi}\left(\mathbb{R}^{n}\right)\|\leq c\| f\right| F_{p(\cdot), q(\cdot)}^{\boldsymbol{w}, \phi}\left(\mathbb{R}^{n}\right)\right\|
$$

with $c$ being a positive constant independent of $f$.

Theorem 4.9 Let $p, q \in \mathcal{P}^{\log }\left(\mathbb{R}^{n}\right), \boldsymbol{w}=\left(w_{j}\right)_{j \in \mathbb{N}_{0}} \in \mathcal{W}_{\alpha_{1}, \alpha_{2}}^{\alpha}\left(\mathbb{R}^{n}\right)$ and $\phi \in \mathcal{G}\left(\mathbb{R}_{+}^{n+1}\right)$.

(i) Let $K, L \geq 0$ with

$$
K>\alpha_{2}+\max \left\{0, \log _{2} \tilde{c}_{1}(\phi)\right\} \quad \text { and } \quad L>\frac{n}{\min \left\{1, p^{-}\right\}}-n-\alpha_{1} .
$$

Suppose that $\left\{a_{Q}\right\}_{Q \in \mathcal{Q}^{*}}$ is a family of $[K, L]$-non-smooth atoms and that $\left\{t_{Q}\right\}_{Q \in \mathcal{Q}^{*}} \in b_{p(\cdot), q(\cdot)}^{w, \phi}\left(\mathbb{R}^{n}\right)$. Then $f:=\sum_{Q \in \mathcal{Q}^{*}} t_{Q} a_{Q}$ converges in $\mathscr{S}\left(\mathbb{R}^{n}\right)$ and

$$
\left\|f\left|B_{p(\cdot), q(\cdot)}^{w, \phi}\left(\mathbb{R}^{n}\right)\|\leq c\| t\right| b_{p(\cdot), q(\cdot)}^{w, \phi}\left(\mathbb{R}^{n}\right)\right\|
$$

with c being a positive constant independent of $t$.

(ii) Conversely, if $f \in B_{p(\cdot), q(\cdot)}^{w, \phi}\left(\mathbb{R}^{n}\right)$, then, for any given $K, L \geq 0$, there exist a sequence $\left\{t_{Q}\right\}_{Q \in \mathcal{Q}^{*}} \in b_{p(\cdot), q(\cdot)}^{w, \phi}\left(\mathbb{R}^{n}\right)$ and a sequence $\left\{a_{Q}\right\}_{Q \in \mathcal{Q}^{*}}$ of $[K, L]-$ nonsmooth atoms such that $f=\sum_{Q \in \mathcal{Q}^{*}} t_{Q} a_{Q}$ in $\mathscr{S}\left(\mathbb{R}^{n}\right)$ and

$$
\left\|t\left|b_{p(\cdot), q(\cdot)}^{\boldsymbol{w}, \boldsymbol{\phi}}\left(\mathbb{R}^{n}\right)\|\leq c\| f\right| B_{p(\cdot), q(\cdot)}^{\boldsymbol{w}, \boldsymbol{(}}\left(\mathbb{R}^{n}\right)\right\|
$$

with c being a positive constant independent of $f$.

Proof Step 1 We start by proving (ii) for what we fix $K, L \geq 0$ and assume that $f \in B_{p(\cdot), q(\cdot)}^{w, \phi}\left(\mathbb{R}^{n}\right)$ is given. Then, by [37, Theorem 5.3], we know that $f$ can be written 
as an atomic decomposition with $\left[K_{1}, L_{1}\right]$-smooth atoms with $K_{1}, L_{1} \in \mathbb{N}_{0}$ chosen so that $K_{1} \geq K$ and $L_{1} \geq L$. Since those atoms are $[K, L]$-non-smooth atoms, cf. Remark 4.5, part (ii) is proved.

Step 2 In this step we show that $f=\sum_{Q \in \mathcal{Q}^{*}} t_{Q} a_{Q}$ converges in $\mathscr{S}\left(\mathbb{R}^{n}\right)$ if $\left\{t_{Q}\right\}_{Q \in \mathcal{Q}^{*}} \in b_{p(\cdot), q(\cdot)}^{\boldsymbol{w}, \phi}\left(\mathbb{R}^{n}\right)$ and $\left\{a_{Q}\right\}_{Q \in \mathcal{Q}^{*}}$ is a family of $[K, L]$-non-smooth atoms with $K, L \geq 0$ such that (4.7) holds. To this end, it suffices to show that

$$
\lim _{N \rightarrow \infty, \Lambda \rightarrow \infty} \sum_{v=0}^{N} \sum_{k \in \mathbb{Z}^{n},|k| \leq \Lambda} t_{Q_{v k}} a_{Q_{v k}}
$$

exists in $\mathscr{S}\left(\mathbb{R}^{n}\right)$, and we rely mainly on the proof of [12, Theorem 4.10$]$, where the corresponding result was proved for the spaces $F_{p(\cdot), q(\cdot)}^{s(\cdot) \phi}\left(\mathbb{R}^{n}\right)$. By (4.7), we know that there exists $r \in\left(0, \min \left\{1, p^{-}, q^{-}\right\}\right)$such that $\alpha_{1}+\frac{n}{p^{-}}(r-1)>-L$. Let

$$
\tilde{p}(x):=\frac{p(x)}{r} \quad \text { and } \quad \tilde{w}_{j}(x):=w_{j}(x) 2^{\frac{n}{p(x)}(r-1)}, \quad \text { for } \text { all } x \in \mathbb{R}^{n}, j \in \mathbb{N}_{0} .
$$

Then $\tilde{\boldsymbol{w}}=\left(\tilde{w}_{j}\right)_{j \in \mathbb{N}_{0}} \in \mathcal{W}_{\tilde{\alpha}_{1}, \tilde{\alpha}_{2}}^{\tilde{\alpha}}\left(\mathbb{R}^{n}\right)$ and

$$
\tilde{\alpha}_{1}:=\alpha_{1}+\frac{n}{p^{-}}(r-1)>-L .
$$

According to [37, Proposition 4.1(i), Remark 4.2(ii)], we have the following sequence of embeddings

$$
b_{p(\cdot), q(\cdot)}^{\boldsymbol{w}, \phi}\left(\mathbb{R}^{n}\right) \hookrightarrow b_{p(\cdot), \infty}^{\boldsymbol{w}, \phi}\left(\mathbb{R}^{n}\right) \hookrightarrow b_{\tilde{p}(\cdot), \infty}^{\tilde{\boldsymbol{w}}, \phi}\left(\mathbb{R}^{n}\right) .
$$

By similar arguments as used in the proof of [12, Theorem 4.10], we conclude that there exist $\delta_{0}>\max \left\{0, \log _{2} \tilde{c}_{1}(\phi)\right\}, a>n$ and $R>0$ as big as we want such that, for all $h \in \mathscr{S}\left(\mathbb{R}^{n}\right)$ and $j \in \mathbb{N}_{0}$,

$$
\begin{aligned}
& \left|\int_{\mathbb{R}^{n}} \sum_{k \in \mathbb{Z}^{n},|k| \leq \Lambda} t_{Q_{v k}} a_{Q_{v k}}(x) h(x) d x\right| \lesssim 2^{-v\left(L+\tilde{\alpha}_{1}\right)} \sum_{j=0}^{\infty} 2^{-j \delta_{0}} \sum_{i=0}^{\infty} 2^{-i(R-a)} . \\
& \cdot \| \sum_{k \in \mathbb{Z}^{n}} \tilde{w}_{v}\left(2^{-v} k\right)\left|t_{Q_{v k}}\right| \chi_{Q_{v k}} \mid L_{\tilde{p}(\cdot)}\left(Q \left(0,2^{\left.\left.i+j+c_{0}\right)\right)} \|\right.\right. \\
& \lesssim 2^{-v\left(L+\tilde{\alpha}_{1}\right)} \sum_{j=0}^{\infty} 2^{-j \delta_{0}} \sum_{i=0}^{\infty} 2^{-i(R+\tilde{\alpha}-a)} \phi\left(Q\left(0,2^{i+j+c_{0}}\right)\right)\left\|t \mid b_{\tilde{p}(\cdot), \infty}^{\tilde{w}, \phi}\left(\mathbb{R}^{n}\right)\right\| \\
& \lesssim 2^{-v\left(L+\tilde{\alpha}_{1}\right)} \sum_{j=0}^{\infty} 2^{-j\left(\delta_{0}-\log _{2} \tilde{c}_{1}(\phi)\right)} \sum_{i=0}^{\infty} 2^{-i\left(R+\tilde{\alpha}-a-\log _{2} \tilde{c}_{1}(\phi)\right)}\left\|t \mid b_{\tilde{p}(\cdot), \infty}^{\tilde{w}, \phi}\left(\mathbb{R}^{n}\right)\right\| \\
& \lesssim 2^{-v\left(L+\tilde{\alpha}_{1}\right)}\left\|t \mid b_{\tilde{p}(\cdot), \infty}^{\tilde{\tilde{w}}, \phi}\left(\mathbb{R}^{n}\right)\right\| .
\end{aligned}
$$

Here we have used the following estimate, coming from the properties of the class $\mathcal{W}_{\tilde{\alpha}_{1}, \tilde{\alpha}_{2}}^{\tilde{\alpha}}\left(\mathbb{R}^{n}\right)$ 


$$
2^{-v \tilde{\alpha}_{1}} \tilde{w}_{\nu}\left(2^{-v} m\right)\left(1+2^{\nu}\left|x-2^{-v} m\right|\right)^{\tilde{\alpha}}(1+|x|)^{\tilde{\alpha}} \gtrsim 2^{-v \tilde{\alpha}_{1}} \tilde{w}_{\nu}(x)(1+|x|)^{\tilde{\alpha}} \gtrsim 1,
$$

where the implicit constants are independent of $x \in \mathbb{R}^{n}, v \in \mathbb{N}_{0}$ and $m \in \mathbb{Z}^{n}$. Therefore, since $L>-\tilde{\alpha}_{1}$, we conclude that the limit of (4.8) exists in $\mathscr{S}\left(\mathbb{R}^{n}\right)$.

Step 3 We deal now with part (i). Firstly, as in the proof of the characterization of $F_{p(\cdot), q(\cdot)}^{s(\cdot)}\left(\mathbb{R}^{n}\right)$ with non-smooth atoms stated in $[12$, Theorem 4.10], we will apply the local means characterization proved in the previous section. Let us assume that $\left\{t_{Q}\right\}_{Q \in \mathcal{Q}^{*}} \in b_{p(\cdot), q(\cdot)}^{w, \phi}\left(\mathbb{R}^{n}\right)$ and that $\left\{a_{Q}\right\}_{Q \in \mathcal{Q}^{*}}$ is a family of $[K, L]$-non-smooth atoms with $K, L \geq 0$ such that (4.7) holds. Since the convergence of $f=\sum_{Q \in \mathcal{Q}^{*}} t_{Q} a_{Q}$ in $\mathscr{S}\left(\mathbb{R}^{n}\right)$ was already shown in the previous step, we are left to the proof of

$$
\left\|f\left|B_{p(\cdot), q(\cdot)}^{\boldsymbol{w}, \phi}\left(\mathbb{R}^{n}\right)\|\lesssim\| t\right| b_{p(\cdot), q(\cdot)}^{\boldsymbol{w}, \phi}\left(\mathbb{R}^{n}\right)\right\| .
$$

Without loss of generality, we may assume that $\left\|t \mid b_{p(\cdot), q(\cdot)}^{w, \phi}\left(\mathbb{R}^{n}\right)\right\|=1$ and show that $\left\|f \mid B_{p(\cdot), q(\cdot)}^{w, \phi}\left(\mathbb{R}^{n}\right)\right\| \lesssim 1$.

Let $k_{j}, j \in \mathbb{N}_{0}$, be local means as in Corollary 3.4. Then,

$$
k_{j} * f=\sum_{v=0}^{j} \sum_{k \in \mathbb{Z}^{n}} t_{Q_{v k}} k_{j} * a_{Q_{v k}}+\sum_{\nu=j+1}^{\infty} \sum_{k \in \mathbb{Z}^{n}} t_{Q_{v k}} k_{j} * a_{Q_{v k}}
$$

and hence

$$
\begin{aligned}
\left\|f \mid B_{p(\cdot), q(\cdot)}^{w, \phi}\left(\mathbb{R}^{n}\right)\right\| \lesssim & \left\|\left(\sum_{v=0}^{j} \sum_{k \in \mathbb{Z}^{n}} w_{j}\left|t_{Q_{v k}}\right|\left|k_{j} * a_{Q_{v k}}\right|\right)_{j \in \mathbb{N}_{0}} \mid \ell_{q(\cdot)}^{\phi}\left(L_{p(\cdot)}\right)\right\|+ \\
& +\left\|\left(\sum_{v=j+1}^{\infty} \sum_{k \in \mathbb{Z}^{n}} w_{j}\left|t_{Q_{v k}}\right|\left|k_{j} * a_{Q_{v k}}\right|\right)_{j \in \mathbb{N}_{0}} \mid \ell_{q(\cdot)}^{\phi}\left(L_{p(\cdot)}\right)\right\| \\
= & : I+I I .
\end{aligned}
$$

In what follows, let $r \in\left(0, \min \left\{1, p^{-}, q^{-}\right\}\right)$such that $L>\frac{n}{r}-n-\alpha_{1}$.

Substep 3.1. Firstly we show that $I \lesssim 1$. For any given $P \in \mathcal{Q}$, we split the sum in $v$ in two parts as follows 


$$
\begin{aligned}
& \frac{1}{\phi(P)}\left\|\left(\sum_{v=0}^{j} \sum_{k \in \mathbb{Z}^{n}} w_{j}\left|t_{Q_{v k}}\right|\left|k_{j} * a_{Q_{v k}}\right|\right)_{j \geq\left(j_{P} \vee 0\right)} \mid \ell_{q(\cdot)}\left(L_{p(\cdot)}(P)\right)\right\| \\
& \lesssim \frac{1}{\phi(P)}\left\|\left(\sum_{v=0}^{\left(j_{P} \vee 0\right)-1} \sum_{k \in \mathbb{Z}^{n}} w_{j}^{r}\left|t_{Q_{v k}}\right|^{r}\left|k_{j} * a_{Q_{v k}}\right|^{r}\right)_{j \geq\left(j_{P} \vee 0\right)} \mid \ell_{\frac{q(\cdot)}{r}\left(L_{\frac{p(\cdot)}{r}}\right)}\right\|^{\frac{1}{r}} \\
& \quad+\frac{1}{\phi(P)}\left\|\left(\sum_{v=\left(j_{P} \vee 0\right)}^{j} \sum_{k \in \mathbb{Z}^{n}} w_{j}^{r}\left|t_{Q_{v k}}\right|^{r}\left|k_{j} * a_{Q_{v k}}\right|^{r}\right)_{j \geq\left(j_{P} \vee 0\right)} \mid \ell_{\frac{q(\cdot)}{r}\left(L_{\frac{p(\cdot)}{r}}\right)}\right\|^{\frac{1}{r}} \\
& =: I_{1}+I_{2},
\end{aligned}
$$

and prove that $I_{i} \lesssim 1$, for $i=1,2$. Observe that $I_{1}=0$ if $j_{P} \leq 0$. Therefore, it suffices to consider the case when $j_{P}>0$. Moreover, note that, by the properties of the admissible weight sequence, the following holds true

$$
w_{j}(x) \lesssim 2^{(j-v) \alpha_{2}} w_{v}\left(2^{-v} k\right)\left(1+2^{v}\left|x-2^{-v} k\right|\right)^{\alpha},
$$

for all $x \in \mathbb{R}^{n}$ and $j \geq v$.

We first estimate $I_{1}$. By (4.11) and Lemma 4.7, we can follow the same procedure as in Step 2 of the proof of [38, Theorem 3.8] and obtain

$$
\begin{aligned}
& \sum_{k \in \mathbb{Z}^{n}} w_{j}(x)^{r}\left|t_{Q_{v k}}\right|^{r}\left|\left(k_{j} * a_{Q_{v k}}\right)(x)\right|^{r} \\
& \lesssim 2^{-(j-v)\left(K-\alpha_{2}\right) r} \sum_{k \in \mathbb{Z}^{n}} w_{\nu}\left(2^{-v} k\right)^{r}\left|t_{Q_{v k}}\right|^{r}\left(1+2^{v}\left|x-2^{v} k\right|\right)^{(\alpha-M) r} \\
& \lesssim 2^{-(j-v)\left(K-\alpha_{2}\right) r} \sum_{i=0}^{\infty} 2^{i(\alpha-M+a) r} \eta_{v, a r} \\
& \quad *\left(\left[\sum_{k \in \mathbb{Z}^{n}} w_{v}\left(2^{-v} k\right)\left|t_{Q_{v k}}\right| \chi_{Q_{v k}} \chi_{Q\left(c_{P}, 2^{\left.i-v+c_{0}\right)}\right.}\right]^{r}\right)(x),
\end{aligned}
$$

where $a>n / r, c_{P}$ is the center of $P$ and $c_{0} \in \mathbb{N}$ independent of $x, P, i, v$ and $k$. Then, by the fact that $\ell_{1}\left(L_{\frac{p(\cdot)}{r}}\right) \hookrightarrow \ell_{\frac{q(\cdot)}{r}}\left(L_{\frac{p(\cdot)}{r}}\right)$ and by Remark 2.7 , we can estimate 


$$
\begin{aligned}
& I_{1} \lesssim \frac{1}{\phi(P)}\left\|\left(\sum_{v=0}^{j_{P}-1} \sum_{k \in \mathbb{Z}^{n}} w_{j}^{r}\left|t_{Q_{v k}}\right|^{r}\left|k_{j} * a_{Q_{v k}}\right|^{r}\right)_{j \geq j_{P}} \mid \ell_{1}\left(L_{\frac{p(\cdot)}{r}}\right)\right\|^{\frac{1}{r}} \\
& =\frac{1}{\phi(P)}\left\{\sum_{j=j_{P}}^{\infty}\left\|\sum_{v=0}^{j_{p}-1} \sum_{k \in \mathbb{Z}^{n}} w_{j}^{r}\left|t_{Q_{v k}}\right|^{r}\left|k_{j} * a_{Q_{v k}}\right|^{r} \mid L_{\frac{p(\cdot)}{r}}\left(\mathbb{R}^{n}\right)\right\|\right\}^{\frac{1}{r}} \\
& \lesssim\left\{\sum_{j=j_{P}}^{\infty} \frac{1}{\phi(P)^{r}} \| \sum_{\nu=0}^{j_{P}-1} 2^{-(j-v)\left(K-\alpha_{2}\right) r} \sum_{i=0}^{\infty} 2^{i(\alpha-M+a) r} \eta_{\nu, a r}\right. \\
& \left.*\left(\left[\sum_{k \in \mathbb{Z}^{n}} w_{v}\left(2^{-v} k\right)\left|t_{Q_{v k}}\right| \chi_{Q_{v k}} \chi_{Q\left(c_{P}, 2^{\left.i-v+c_{0}\right)}\right.}\right]^{r}\right) \mid L_{\frac{p(\cdot)}{r}}\left(\mathbb{R}^{n}\right) \|\right\}^{1 / r} \\
& \lesssim\left\{\sum_{j=j_{P}}^{\infty} \frac{1}{[\phi(P)]^{r}} \sum_{\nu=0}^{j_{P}-1} 2^{-(j-\imath)\left(K-\alpha_{2}\right) r} \sum_{i=0}^{\infty} 2^{i(\alpha-M+a) r}\right. \\
& \left.\cdot\left\|\sum_{k \in \mathbb{Z}^{n}} w_{v}\left(2^{-v} k\right)\left|t_{Q_{v k}}\right| \chi_{Q_{v k}} \mid L_{p(\cdot)}\left(Q\left(c_{P}, 2^{i-v+c_{0}}\right)\right)\right\|^{r}\right\}^{1 / r} \\
& \lesssim\left\{\sum_{j=j_{P}}^{\infty} \sum_{\nu=0}^{j_{P}-1} 2^{-(j-\imath)\left(K-\alpha_{2}\right) r} \sum_{i=0}^{\infty} 2^{i(\alpha-M+a) r} \frac{\phi\left(Q\left(c_{P}, 2^{i-v+c_{0}}\right)\right)^{r}}{[\phi(P)]^{r}}\right\}^{1 / r} \\
& \lesssim\left\{\sum_{j=j_{P}}^{\infty} \sum_{\nu=0}^{j_{P}-1} 2^{-(j-\nu)\left(K-\alpha_{2}\right) r} \sum_{i=0}^{\infty} 2^{i(\alpha-M+a) r} 2^{\left(i-\nu+j_{P}\right) r \log _{2} \tilde{c}_{1}(\phi)}\right\}^{1 / r}
\end{aligned}
$$

where we have used (2.5) in the last step. Now, noting that $K>\alpha_{2}+\max \left\{0, \log _{2} \tilde{c}_{1}(\phi)\right\}$, we conclude that

$$
I_{1} \lesssim\left\{\sum_{j=j_{P}}^{\infty} 2^{j_{P} \log _{2} \tilde{c}_{1}(\phi)} 2^{-j\left(K-\alpha_{2}\right) r} \sum_{\nu=0}^{j_{P}-1} 2^{v\left(K-\alpha_{2}-\log _{2} \tilde{c}_{1}(\phi)\right) r}\right\}^{1 / r} \lesssim 1,
$$

where $M$ is chosen large enough such that $M>\alpha+a+\log _{2} \tilde{c}_{1}(\phi)$.

Let us prove now that $I_{2} \lesssim 1$. Here we follow Step 3 of the proof of [12, Theorem 4.10] and use the estimate (4.11) to obtain that, for fixed $x \in P, j \geq\left(j_{P} \vee 0\right)$ and $v$ with $\left(j_{P} \vee 0\right) \leq v \leq j$ 


$$
\begin{aligned}
& \sum_{k \in \mathbb{Z}^{n}} w_{j}(x)^{r}\left|t_{Q_{v k}}\right|^{r}\left|\left(k_{j} * a_{Q_{v k}}\right)(x)\right|^{r} \\
& \lesssim \sum_{k \in \mathbb{Z}^{n}} w_{j}(x)^{r}\left|t_{Q_{v k}}\right|^{r} 2^{-(j-v)\left(K-\alpha_{2}\right) r}\left(1+2^{v}\left|x-x_{Q_{v k}}\right|\right)^{(\alpha-M) r} \\
& \lesssim 2^{-(j-\nu)\left(K-\alpha_{2}\right) r} \sum_{i=0}^{\infty} 2^{(j+i)(\alpha-M+a) r} \eta_{v, a r} * \\
& *\left(\left[\sum_{k \in \mathbb{Z}^{n}}\left|t_{Q_{v k}}\right| w_{v}\left(2^{-v} k\right) \chi_{Q_{v k}} \chi_{Q\left(c_{p}, 2^{\left.i+j-j p+c_{0}\right)}\right.}\right]^{r}\right)(x),
\end{aligned}
$$

where $a>n / r, c_{P}$ is the center of $P, c_{0} \in \mathbb{N}$ is a positive constant independent of $x, P, i, v, k, j$. Similarly as above, we have

$$
\begin{aligned}
& I_{2}=\frac{1}{\phi(P)}\left\{\sum_{j=\left(j_{P} \vee 0\right)}^{\infty}\left\|\sum_{v=\left(j_{P} \vee 0\right)}^{j} \sum_{k \in \mathbb{Z}^{n}} w_{j}^{r}\left|t_{Q_{v k}}\right|^{r}\left|k_{j} * a_{Q_{v k}}\right|^{r} \mid L_{\frac{p(\cdot)}{r}}\left(\mathbb{R}^{n}\right)\right\|\right\}^{\frac{1}{r}} \\
& \lesssim\left\{\sum_{j=\left(j_{P} \vee 0\right)}^{\infty} \frac{1}{\phi(P)^{r}} \| \sum_{\nu=\left(j_{P} \vee 0\right)}^{j} 2^{-(j-\nu)\left(K-\alpha_{2}\right) r} \sum_{i=0}^{\infty} 2^{(j+i)(\alpha-M+a) r} \eta_{\nu, a r} *\right. \\
& \left.*\left(\left[\sum_{k \in \mathbb{Z}^{n}} w_{v}\left(2^{-v} k\right)\left|t_{Q_{v k}}\right| \chi_{Q_{v k}} \chi_{Q\left(c_{p}, 2^{\left.i+j-j p+c_{0}\right)}\right.}\right]^{r}\right) \mid L_{\frac{p(\cdot)}{r}}\left(\mathbb{R}^{n}\right) \|\right\}^{1 / r} \\
& \lesssim\left\{\sum_{j=\left(j_{P} \vee 0\right)}^{\infty} \frac{1}{[\phi(P)]^{r}} \sum_{\nu=\left(j_{P} \vee 0\right)}^{j} 2^{-(j-\imath)\left(K-\alpha_{2}\right) r} \sum_{i=0}^{\infty} 2^{(i+j)(\alpha-M+a) r} .\right. \\
& \left.\cdot\left\|\sum_{k \in \mathbb{Z}^{n}} w_{\nu}\left(2^{-v} k\right)\left|t_{Q_{v k}}\right| \chi_{Q_{v k}} \mid L_{p(\cdot)}\left(Q\left(c_{P}, 2^{i+j-j_{P}+c_{0}}\right)\right)\right\|^{r}\right\}^{1 / r} \\
& \lesssim\left\{\sum_{j=\left(j_{P} \vee 0\right)}^{\infty} \sum_{\nu=\left(j_{P} \vee 0\right)}^{j} 2^{-(j-v)\left(K-\alpha_{2}\right) r} .\right. \\
& \left.\cdot \sum_{i=0}^{\infty} 2^{(i+j)(\alpha-M+a) r} \frac{\phi\left(Q\left(c_{P}, 2^{i+j-j_{P}+c_{0}}\right)\right)^{r}}{[\phi(P)]^{r}}\right\}^{1 / r} \\
& \lesssim\left\{\sum_{j=\left(j_{P} \vee 0\right)}^{\infty} \sum_{\nu=\left(j_{P} \vee 0\right)}^{j} 2^{-(j-\imath)\left(K-\alpha_{2}\right) r} \sum_{i=0}^{\infty} 2^{(i+j)(\alpha-M+a) r} 2^{(i+j) r \log _{2} \tilde{c}_{1}(\phi)}\right\}^{1 / r},
\end{aligned}
$$

Choosing $\quad M$ large enough such that $M>\alpha+a+\log _{2} \tilde{c}_{1}(\phi)$ and since $K>\alpha_{2}+\max \left\{0, \log _{2} \tilde{c}_{1}(\phi)\right\}$, we conclude that

$$
I_{2} \lesssim\left\{\sum_{j=\left(j_{P} \vee 0\right)}^{\infty} 2^{-j\left(K-\alpha_{2}-\log _{2} \tilde{c}_{1}(\phi)-\alpha+M-a\right) r} \sum_{\nu=\left(j_{P} \vee 0\right)}^{j} 2^{\nu\left(K-\alpha_{2}\right) r}\right\}^{1 / r} \lesssim 1,
$$


as we wanted to demonstrate.

Substep 3.2 Lastly, we will show $I I \lesssim 1$. For $v>j$, by the properties of the admissible weight sequence we have, for all $x \in \mathbb{R}^{n}$,

$$
w_{j}(x) \lesssim 2^{-(\nu-j) \alpha_{1}} w_{\nu}\left(2^{-v} k\right)\left(1+2^{j}\left|x-2^{-v} k\right|\right)^{\alpha} .
$$

Together with Lemma 4.7, this leads us to

$$
\begin{aligned}
& \sum_{k \in \mathbb{Z}^{n}} w_{j}(x)^{r}\left|t_{Q_{v k}}\right|^{r}\left|\left(k_{j} * a_{Q_{v k}}\right)(x)\right|^{r} \\
& \lesssim 2^{-(\nu-j)\left(L+n+\alpha_{1}-\frac{n}{r}\right) r} \sum_{k \in \mathbb{Z}^{n}} w_{v}\left(2^{-v} k\right)^{r}\left|t_{Q_{v k}}\right|^{r}\left(1+2^{j}\left|x-2^{v} k\right|\right)^{(\alpha-M) r} \\
& \lesssim 2^{-(\nu-j)\left(L+n+\alpha_{1}\right) r} \sum_{i=0}^{\infty} 2^{i(\alpha-M+a) r} \eta_{j, a r} * \\
& \quad *\left(\left[\sum_{k \in \mathbb{Z}^{n}} w_{v}\left(2^{-v} k\right)\left|t_{Q_{v k}}\right| \chi_{Q_{v k}} \chi_{Q\left(c_{P}, 2^{\left.i-j_{P}+c_{0}\right)}\right.}\right]^{r}\right)(x)
\end{aligned}
$$

where $a>n / r, c_{P}$ is the center of $P$ and $c_{0} \in \mathbb{N}$ independent of $x, P, i, v$ and $k$. We go back to $I I$ and, by Lemmas 2.12 and 2.10 together with our assumptions, we conclude

$$
\begin{aligned}
& I I=\left\|\left\{\sum_{v=j+1}^{\infty} \sum_{k \in \mathbb{Z}^{n}} w_{j}(x)^{r}\left|t_{Q_{v k}}\right|^{r}\left|\left(k_{j} * a_{Q_{v k}}\right)(x)\right|^{r}\right\}_{j \in \mathbb{N}_{0}} \mid \ell_{\frac{q(j)}{r}}^{\phi}\left(L_{\frac{p(-)}{r}}\right)\right\|^{\frac{1}{r}} \\
& \lesssim\left\{\sum_{i=0}^{\infty} 2^{i(\alpha-M+a) r} \|\left\{\sum_{v=j+1}^{\infty} 2^{-(v-j)\left(L+n+\alpha_{1}-\frac{n}{r}\right) r} \eta_{j, a r} *\right.\right. \\
& \left.*\left(\left[\sum_{k \in \mathbb{Z}^{n}} w_{\nu}\left(2^{-v} k\right)\left|t_{Q_{\nu k}}\right| \chi_{Q_{\nu k}} \chi_{Q\left(c_{p}, 2^{\left.i-j p p+c_{0}\right)}\right.}\right]^{r}\right)\right\}_{j \in \mathbb{N}_{0}} \mid \ell_{\frac{q(\cdot)}{r}}^{\phi}\left(L_{\left.\frac{p(-)}{r}\right)}||\right\}^{\frac{1}{r}} \\
& \lesssim\left\{\sum_{i=0}^{\infty} 2^{i(\alpha-M+a) r} \|\left\{\eta_{\nu, a r} *\left(\left[\sum_{k \in \mathbb{Z}^{n}} w_{\nu}\left(2^{-v} k\right)\left|t_{Q_{\nu k}}\right| \chi_{Q_{\nu k}} \chi_{Q\left(c_{p}, 2^{\left.i-j p+\epsilon_{0}\right)}\right.}\right]^{r}\right)\right\}_{j \in \mathbb{N}_{0}} \mid \ell_{\frac{q(v)}{r}}^{\phi}\left(L_{\frac{p(v)}{r}} \|\right\}^{\frac{1}{r}}\right. \\
& \lesssim\left\{\sum_{i=0}^{\infty} 2^{i(\alpha-M+a) r} \cdot\left\|\left\{\sum_{k \in \mathbb{Z}^{n}} w_{\nu}\left(2^{-v} k\right)\left|t_{Q_{v k}}\right| \chi_{Q_{v k}} \chi_{Q\left(c_{p}, 2^{\left.i-j p+c_{0}\right)}\right.}\right\}_{j \in \mathbb{N}_{0}} \mid \ell_{q(\cdot)}^{\phi}\left(L_{p(\cdot)}\right)\right\|\right\}^{\frac{1}{r}} \\
& \lesssim\left\{\sum_{i=0}^{\infty} 2^{i\left(\alpha-M+a+\log _{2} \tilde{c}_{1}(\phi)\right) r}\left\|t \mid b_{p(\cdot), q(\cdot)}^{w, \phi}\left(\mathbb{R}^{n}\right)\right\|^{r}\right\}^{\frac{1}{r}} \\
& \lesssim 1 \text {. }
\end{aligned}
$$

Here $M$ is chosen large enough such that $M>\alpha+a+\log _{2} \tilde{c}_{1}(\phi)$. We have then finished the proof. 


\section{Pointwise multipliers}

Following [30] and also the corresponding results in the variable setting in [10, 12], in this section we aim to provide a result on pointwise multipliers for the spaces under consideration. More precisely, let $\varphi$ be a bounded function on $\mathbb{R}^{n}$. The question is under which conditions the mapping $f \mapsto \varphi \cdot f$ makes sense and generates a bounded operator in a given space $B_{p(\cdot), q(\cdot)}^{w, \phi}\left(\mathbb{R}^{n}\right)$ or $F_{p(\cdot), q(\cdot)}^{\boldsymbol{w}, \phi}\left(\mathbb{R}^{n}\right)$. This can be answered in a very pleasant way using the non-smooth atomic decomposition from the previous section. We point out that, although it is a very important tool in the study of function spaces, the smooth atomic decomposition is not helpful in this case. The reason is that the moment conditions like (4.2) are, in general, destroyed by multiplication with $\varphi$. For more references on the topic, we refer to [12].

We start by presenting two helpful results proved in [30]. The first shows that the product of two functions in $\mathscr{C}\left(\mathbb{R}^{n}\right)$ is still a function in this space, as in [30, Lemma 4.2]. In the second lemma is stated that the product of a $[K, L]$-non-smooth atom with a function $\varphi \in \mathscr{C}^{p}\left(\mathbb{R}^{n}\right)$ is still a $[K, L]$-non-smooth atom, and represents a slight normalization of [30, Lemma 4.3].

Lemma 5.1 Let $s \geq 0$. There exists a constant $c>0$ such that for all $f, g \in \mathscr{C}^{s}\left(\mathbb{R}^{n}\right)$, the product $f \cdot g$ belongs to $\mathscr{C}\left(\mathbb{R}^{n}\right)$ and it holds

$$
\left\|f \cdot g\left|\mathscr{C}^{S}\left(\mathbb{R}^{n}\right)\|\leq c\| f\right| \mathscr{C}^{S}\left(\mathbb{R}^{n}\right)\right\| \cdot\left\|g \mid \mathscr{C}^{S}\left(\mathbb{R}^{n}\right)\right\| .
$$

Lemma 5.2 There exists a constant $c$ with the following property: for all $\nu \in \mathbb{N}_{0}$, $m \in \mathbb{Z}^{n}$, all $[K, L]$-non-smooth atoms $a_{v, m}$ with support in $3 Q_{v, m}$ and all $\varphi \in \mathscr{C}^{p}\left(\mathbb{R}^{n}\right)$ with $\rho \geq \max \{K, L\}$, the product

$$
c\left\|\varphi \mid \mathscr{C}^{p}\left(\mathbb{R}^{n}\right)\right\|^{-1} \cdot \varphi \cdot a_{v, m}
$$

is a $[K, L]$-non-smooth atom with support in $3 Q_{v, m}$.

The main result of this section can now be presented. We skip the proof, once it can be carried out as the proof of [10, Theorem 4.3].

Theorem 5.3 Let $p, q, s, \phi$ as in Definition 2.16.

(i) Let

$$
\rho>\max \left\{\alpha_{2}, \alpha_{2}+\log _{2} \tilde{c}_{1}(\phi), \frac{n}{\min \left\{1, p^{-}\right\}}-n-\alpha_{1}\right\} .
$$

Then there exists a positive number $c$ such that

$$
\begin{aligned}
& \left\|\varphi \cdot f\left|B_{p(\cdot), q(\cdot)}^{w, \phi}\left(\mathbb{R}^{n}\right)\|\leq c\| \varphi\right| \mathscr{C}^{\rho}\left(\mathbb{R}^{n}\right)\right\| \cdot\left\|f \mid B_{p(\cdot), q(\cdot)}^{w, \phi}\left(\mathbb{R}^{n}\right)\right\| \\
& \text { for all } \varphi \in \mathscr{C}^{p}\left(\mathbb{R}^{n}\right) \text { and all } f \in B_{p(\cdot), q(\cdot)}^{w, \phi}\left(\mathbb{R}^{n}\right) \text {. }
\end{aligned}
$$


(ii) Let

$$
\rho>\max \left\{\alpha_{2}, \alpha_{2}+\log _{2} \tilde{c}_{1}(\phi), \frac{n}{\min \left\{1, p^{-}, q^{-}\right\}}-n-\alpha_{1}\right\} .
$$

\section{Then there exists a positive number $c$ such that}

$$
\begin{aligned}
& \left\|\varphi \cdot f\left|F_{p(\cdot), q(\cdot)}^{w, \phi}\left(\mathbb{R}^{n}\right)\|\leq c\| \varphi\right| \mathscr{C}^{p}\left(\mathbb{R}^{n}\right)\right\| \cdot\left\|f \mid F_{p(\cdot), q(\cdot)}^{w, \phi}\left(\mathbb{R}^{n}\right)\right\| \\
& \text { for all } \varphi \in \mathscr{C}^{p}\left(\mathbb{R}^{n}\right) \text { and all } f \in F_{p(\cdot), q(\cdot)}^{w, \phi}\left(\mathbb{R}^{n}\right) \text {. }
\end{aligned}
$$

Acknowledgements The author was supported by the German Research foundation (DFG), Grant no. Ha 2794/8-11.

Funding Open Access funding enabled and organized by Projekt DEAL.

Open Access This article is licensed under a Creative Commons Attribution 4.0 International License, which permits use, sharing, adaptation, distribution and reproduction in any medium or format, as long as you give appropriate credit to the original author(s) and the source, provide a link to the Creative Commons licence, and indicate if changes were made. The images or other third party material in this article are included in the article's Creative Commons licence, unless indicated otherwise in a credit line to the material. If material is not included in the article's Creative Commons licence and your intended use is not permitted by statutory regulation or exceeds the permitted use, you will need to obtain permission directly from the copyright holder. To view a copy of this licence, visit http://creativecommons.org/licen ses/by/4.0/.

\section{References}

1. Almeida, A., Caetano, A.: On 2-microlocal spaces with all exponents variable. Nonlinear Anal. 135, 97-119 (2016)

2. Almeida, A., Caetano, A.: Atomic and molecular decompositions in variable exponent 2-microlocal spaces and applications. J. Funct. Anal. 270, 1888-1921 (2016)

3. Almeida, A., Hästö, P.: Besov spaces with variable smoothness and integrability. J. Funct. Anal. 258(5), 1628-1655 (2010)

4. Bony, J.M.: Second microlocalization and propagation of singularities for semilinear hyperbolic equations. Hyperbolic equations and related topics (Katata/Kyoto, 1984), pp. 11-49. Academic Press, New York (1986)

5. Diening, L.: Maximal function on generalized Lebesgue spaces $\$ \mathrm{~L}^{\wedge}\{\mathrm{p}($ lcdot $)\} \$$. Math. Inequal. Appl. 7(2), 245-253 (2004)

6. Diening, L., Hästö, P., Roudenko, S.: Function spaces of variable smoothness and integrability. J. Funct. Anal. 256(6), 1731-1768 (2009)

7. Diening, L., Harjuletho, P., Hästö, P., Růžička, M.: Lebesgue and Sobolev Spaces with Variable Exponents. Springer, New York (2011)

8. Drihem, D.: Atomic decomposition of Besov-type and Triebel-lizorkin-type spaces. Sci. China Math. 56, 1073-1086 (2013)

9. Edmunds, D.E., Rákosník, J.: Sobolev embeddings with variable exponent. Stud. Math. 143(3), 267-293 (2000)

10. Gonçalves, H.F., Kempka, H.: Non-smooth atomic decomposition of 2-microlocal spaces and application to pointwise multipliers. J. Math. Anal. Appl. 434, 1875-1890 (2016)

11. Gonçalves, H.F., Kempka, H.: Intrinsic atomic characterization of 2-microlocal spaces with variable exponents on domains. Rev. Mat. Complut. 30(3), 467-486 (2017) 
12. Gonçalves, H.F., Moura, S.D.: Characterization of Triebel-Lizorkin type spaces with variable exponents via maximal functions, local means and non-smooth atomic decompositions. Math. Nachr. 291, 2024-2044 (2018). https://doi.org/10.1002/mana.201700257

13. Gonçalves, H.F., Moura, S.D., Neves, J.S.: On trace spaces of 2-microlocal spaces. J. Funct. Anal. 267, 3444-3468 (2014)

14. Gonçalves, H.F., Kempka, H., Vybíral, J.: Franke-Jawerth embeddings for Besov and TriebelLizorkin spaces with variable exponents. Ann. Acad. Sci. Fenn. Math. 43, 1-23 (2018)

15. Kempka, H.: Generalized \$2\$-microlocal Besov spaces. Ph.D. thesis, University of Jena, Germany (2008)

16. Kempka, H.: 2-Microlocal Besov and Triebel-Lizorkin spaces of variable integrability. Rev. Mat. Complut. 22, 227-251 (2009)

17. Kempka, H.: Atomic, molecular and wavelet decomposition of 2-microlocal Besov and TriebelLizorkin spaces with variable integrability. Funct. Approx. Comment. Math. 43, 171-208 (2010)

18. Kempka, H.: Intrinsic characterization and the extension operator in variable exponent function spaces on special Lipschitz domains. In: Jain, P., Schmeisser, H-J (eds.) Function spaces and inequalities, New Delhi, India, December 11-15, 2015. Singapore: Springer. Springer Proceedings in Mathematics and Statistics 206, 175-192 (2017). https://doi.org/10.1007/978-981-10-6119-6_8

19. Kempka, H., Vybíral, J.: Spaces of variable smoothness and integrability: Characterisations by local means and ball means of differences. J. Fourier Anal. Appl. 18(4), 852-891 (2012)

20. Kempka, H., Vybíral, J.: A note on the spaces of variable integrability and summability of Almeida and Hästö. Proc. Am. Math. Soc. 141(9), 3207-3212 (2013)

21. Kováčik, O., Rákosník, J.: On spaces $\$ \mathrm{~L}^{\wedge}\{\mathrm{p}(\mathrm{x})\} \$$ and $\$ \mathrm{~W}^{\wedge}\{\mathrm{k}, \mathrm{p}(\mathrm{x})\} \$$. Czechoslov. Math. J. 40(116), 592-618 (1991)

22. Liang, Y., Yang, D., Yuan, W., Sawano, Y., Ullrich, T.: A new framework for generalized Besovtype and Triebel-Lizorkin type spaces. Diss. Math. (Rozprawy Mat.) 489, 1-114 (2013)

23. Moura, S.D., Neves, J.S., Schneider, C.: On trace spaces of 2-microlocal Besov spaces with variable integrability. Math. Nachr. 286(11-12), 1240-1254 (2013)

24. Nakai, E.: Pointwise multipliers for functions of weighted bounded mean oscillation. Stud. Math. 105(2), 105-119 (1993)

25. Nakai, E.: The Campanato, Morrey and Hölder spaces on spaces of homogeneous type. Stud. Math. 176(1), 1-19 (2006)

26. Nakai, E., Sawano, Y.: Hardy spaces with variable exponents and generalized Campanato spaces. J. Funct. Anal. 262(9), 3665-3748 (2012)

27. Orlicz, W.: Über konjugierte Exponentenfolgen. Stud. Math. 3, 200-212 (1931)

28. Peetre, J.: On spaces of Triebel-Lizorkin type. Ark. Mat. 13, 123-130 (1975)

29. Rychkov, V.S.: On a theorem of Bui, Paluszyski, and Taibleson. Proc. Steklov Inst. Math. 227, 280292 (1999)

30. Scharf, B.: Atomic representations in function spaces and applications to pointwise multipliers and diffeomorphisms, a new approach. Math. Nachr. 286(2-3), 283-305 (2013)

31. Tang, L., Xu, J.: Some properties of Morrey type Besov-Triebel spaces. Math. Nachr. 278, 904-917 (2005)

32. Triebel, H.: Fractals and Spectra. Birkhäuser, Basel (1997)

33. Triebel, H.: Local Function Spaces, Heat and Navier-Stokes Equations. EMS Tracts in Mathematics, vol. 20. European Mathematical Society (EMS), Zurich (2013)

34. Triebel, H.: Hybrid Function Spaces, Heat and Navier-Stokes Equations. EMS Tracts in Mathematics, vol. 24. European Mathematical Society (EMS), Zurich (2015)

35. Triebel, H., Winkelvoß, H.: Intrinsic atomic characterization of function spaces on domains. Math. Z. 221(1), 647-673 (1996)

36. Vybíral, J.: Sobolev and Jawerth embeddings for spaces with variable smoothness and integrability. Ann. Acad. Sci. Fenn. Math. 34(2), 529-544 (2009)

37. Wu, S., Yang, D., Yuan, W., Zhuo, C.: Variable 2-microlocal Besov-Triebel-Lizorkin-type spaces. Acta. Math. Sin. Engl. Ser. 34, 699-748 (2018). https://doi.org/10.1007/s10114-018-7311-7

38. Yang, D., Zhuo, C., Yuan, W.: Triebel-Lizorkin type spaces with variable exponent. Banach J. Math. Anal. 9(4), 146-202 (2015)

39. Yang, D., Zhuo, C., Yuan, W.: Besov-type spaces with variable smoothness and integrability. J. Funct. Anal. 269, 1840-1898 (2015)

40. Yuan, W., Sickel, W., Yang, D.: Morrey and Campanato Meet Besov, Lizorkin and Triebel. Lecture Notes in Mathematics, vol. 2005. Springer, Berlin (2010) 
41. Yuan, W., Yang, D.: Characterizations of Besov-type and Triebel-Lizorkin-type spaces via maximal functions and local means. Nonlinear Anal. 73, 3805-3820 (2010)

42. Zhuo, C., Chang, D., Yang, D.: Ball average characterizations of variable Besov-type spaces. Taiwan. J. Math. 23, 427-452 (2019) 